\title{
ON THE THEORY OF DIFFERENTIAL FORMS ON ALGEBRAIC VARIETIES
}

\author{
YÛSAKU KAWAHARA
}

Let $K$ be a function field of one variable over a perfect field $k$ and let $v$ be a valuation of $K$ over $k$. Then $v(d x)=v\left(\mathscr{D}_{x}\right)-v(x)_{\infty}$, where $\mathscr{D}_{x}$ is the different-divisor (Verzweigungsdivisor) of $K / k(x)$, and $(x)_{\infty}$ is the denominatordivisor (Nennerdivisor) of $x$. In $\S 1$ we consider a generalization of this theorem in the function fields of many variables under some conditions. In $\S 2$ and $\S 3$ we consider the differential forms of the first kind on algebraic varieties, or the differential forms which are finite at every simple point of normal varieties and subadjoint hypersurfaces which are developed by Clebsch and Picard in the classical case. In $\S 4$ we give a proof of the following theorem. ${ }^{1)}$ Let $V^{r}$ be a normal projective variety defined over a field $k$ of characteristic 0 , and let $\omega_{1}, \ldots, \omega_{s}$ be linearly independent simple closed differential forms which are finite at every simple point of $V^{r}$. Then the induced forms on a generic hyperplane section are also linearly independent.

I express my hearty thanks to Mr. Y. Nakai for his useful remarks.

$\S 1$. Let $K$ be a field, generated over a field $k$ by a set of quantities and let $K$ be of dimension $n$ over $k$. If $K$ is separably algebraic over $k\left(x_{1}, \ldots, x_{n}\right)$ where $x_{1}, \ldots, x_{n}$ is a set of algebraically independent quantities in $K$ over $k$, we say that $x_{1}, \ldots, x_{n}$ are separating generators of $K$ over $k$. Every differential form belonging to the extension $K$ over $k$ is expressed in one and only one way as a polynomial in $d x_{1}, \ldots, d x_{n}$ with coefficients in $K$.

Lemma 1. Let $K$ be a separably generated n-dimensional extension of $k$. Then $n$ differentials $d x_{1}, \ldots, d x_{n}$ of $x_{1}, \ldots, x_{n}$ in $K$ are linearly independent over $K$ if and only if $x_{1}, \ldots, x_{n}$ are separating generators of $K$ over $k$.

Proof. If $d x_{1}, \ldots, d x_{n}$ are linearly independent over $K$, we get for all $z$ in $K$

$$
d z=\sum_{i=1}^{n} a_{i}(z) d x_{i}, \quad a_{i}(z) \in K
$$

Received January 23, 1956.

1) When $V$ is without singularity, this theorem is well known, see J. Igusa [4]. 
Therefore $n$ derivations $D_{i}$ of $K$ defined by $D_{t}(z)=a_{i}(z)$, form a base of the module of all the derivations of $K$ over $k$. It follows immediately that every derivation of $K$ over $k$ does not annul all the elements of $k\left(x_{1}, \ldots, x_{n}\right)$. Therefore by F-I, Th. $1,{ }^{2)} K / k\left(x_{1}, \ldots, x_{n}\right)$ is separably algebraic.

Lemma 2. Let $x_{1}, \ldots, x_{n}$ be separating generators, and

$$
d z_{1} \ldots d z_{s}=\sum_{i_{1}<\ldots<i_{s}} a_{i_{1} \ldots i_{s}} d x_{i_{1}} \ldots d x_{i_{s}}, \quad a_{i_{1} \ldots i_{s}} \in K .
$$

Then $a_{i_{1} \ldots i_{s}} \neq 0$ if and only if $\left(z_{1}, \ldots, z_{s}, x_{1}, \ldots \hat{x}_{i_{1}} \ldots \hat{x}_{i_{s}} \ldots, x_{n}\right)$ are separating generators of $K$ over $k$.

Proof. This follows immediately from Lemma 1.

Let $K$ be a regular $n$-dimensional extension of $k$. In $K$ we consider an $(n-1)$-dimensional valuation $v$. When $x_{1}, \ldots, x_{n}$ are separating generators of $K$ over $k$ we associate a number $v\left(\mathfrak{D}_{x_{1} \ldots x_{n}}\right)$ with $v$ in a similar way as in the case of dimension 1 . Namely, let $\mathfrak{0}$ be the set of all the elements $z$ of $k\left(x_{1}, \ldots, x_{n}\right)$ such that $v(z) \geqslant 0$, and let $\overline{\mathfrak{D}}$ be the set of all the elements in $K$ which are integral over $D$. Then the different-ideal of $\bar{D}$ with respect to $D$ is a principal ideal $(\varphi)$ in $\overline{\mathfrak{D}}$. We define $v\left(\mathscr{D}_{x_{1} \ldots x_{n}}\right)$ by

$$
v\left(\mathfrak{D}_{x_{1} \ldots x_{n}}\right)=v(\varphi) .
$$

More generally, if $K$ is separably algebraic over a subfield $K_{0}$, then we can define $v\left(\mathfrak{D}_{K / K_{0}}\right)$ similarly. $v\left(\mathfrak{D}_{x_{1} \ldots x_{n}}\right)$ may be $\neq 0$ for infinitely many $v$, but if we treat only the valuations $v_{W}$ in $K$ with respect to the subvarieties $W^{n-1}$ of a normal model $V^{n}$ of $K$, then $v_{W}\left(\mathscr{D}_{x_{1} \ldots x_{u}}\right) \neq 0$ for a finite number of $v_{W}$. The following lemma is well known.

Lemma 3. ${ }^{3 !} \quad$ Let $K$ be a regular 1-dimensional extension over $k$, and let $v$ be a valuation of $K$. Then if $x$ and $z$ are the elements in $K$ such that $K / k(x)$ and $K / k(z)$ are separably algebraic, we get

$$
v\left(\frac{d z}{d x}\right)=v\left(\mathfrak{D}_{z}\right)-v\left(\mathfrak{D}_{x}\right)+2 v(x)_{\infty}-2 v(z)_{\infty} .
$$

where $v(x)_{\infty}=0$ if $v(x) \geqslant 0, v(x)_{\infty}=-v(x)$ if $v(x)<0$.

Lemma 4. Let $K$ be a regular $n$-dimensional extension over $k$ and let

2) A. Weil [11] Chapter I, Th. 1, noted by F-I, Th. 1.

3) J. Weissinger [12]. 
$x_{1}, \ldots, x_{n}$ be separating generators of $K$ and

$$
d z=P_{1} d x_{1}+\ldots+P_{n} d x_{n}
$$

Let $v$ be an $(n-1)$-dimensional valuation such that $v\left(x_{i}\right) \geqslant 0(i=1, \ldots, n-1)$ and let $\bar{x}_{i}(i=1, \ldots, n-1)$ be the residue class mod $v$ which contains $x_{i}$. Then, if $\bar{x}_{1}, \ldots, \bar{x}_{n-1}$ are algebraically independent in the residue class field of $v$ over $k$ and $P_{n} \neq 0$, we have

$$
v\left(P_{n}\right)=v\left(\mathscr{D}_{x_{1} \ldots x_{n-1} z}\right)-v\left(\mathfrak{D}_{x_{1}} \ldots x_{x_{1}-1} x_{n}\right)-2 v(z)_{\infty}+2 v(x)_{\infty} .
$$

Proof. Let $k\left(x_{1}, \ldots, x_{n-1}\right) *$ be the algebraic closure of $k\left(x_{1}, \ldots, x_{n-1}\right)$ in $K$. Since $\bar{x}_{1}, \ldots, \bar{x}_{n-1}$ are algebraically independent, we can consider $v$ as a valuation of $K / k\left(x_{1}, \ldots, x_{n-1}\right)^{*} ; K$ is of dimension 1 over $k\left(x_{1}, \ldots, x_{n-1}\right)^{*}$. If we express the differential belonging to the extension $K$ of $k\left(x_{1}, \ldots, x_{n-1}\right)^{*}$ with $d^{\prime}$, then $d^{\prime} z=P_{n} d^{\prime} x_{n}$. Therefore, from Lemma 3,

$$
v\left(P_{n}\right)=v\left(\mathfrak{D}_{z}^{\prime}\right)-v\left(\mathfrak{D}_{x_{n}}^{\prime}\right)-2 v(z)_{\infty}+2 v(x)_{\infty},
$$

where $\mathfrak{D}_{z}^{\prime}$ and $\mathfrak{D}_{x_{n}}^{\prime}$ are the different-divisors with respect to $K / k\left(x_{1}, \ldots, x_{n-1}\right)^{*}(z)$ and $K / k\left(x_{1}, \ldots, x_{n-1}\right)^{*}\left(x_{n}\right)$ respectively. But since $v\left(\mathfrak{D}_{z}^{\prime}\right)=v\left(\mathfrak{D}_{x_{1} \ldots x_{n-1} z}\right)$, $v\left(\mathfrak{D}_{x_{n}}^{\prime}\right)=v\left(\mathfrak{D}_{x_{1} \ldots x_{n}}\right)$, we have

$$
v\left(P_{n}\right)=v\left(\mathfrak{D}_{x_{1} \ldots x_{k-1} z}\right)-v\left(\mathfrak{D}_{x_{1} \ldots x_{n}}\right)-2 v(z)_{\infty}+2 v\left(x_{n}\right)_{\infty} .
$$

THEOREM 1. Let $K$ be a regular $n$-dimensional extension over $k$, and let $x_{1}, \ldots, x_{n}$ be separating generators of $K$ and

$$
d u_{1} \ldots d u_{n}=R d x_{1} \ldots d x_{n} .
$$

Let $v$ be an $(n-1)$-dimensional valuation of $K$. Suppose that $n-1$ elements among $u_{1}, \ldots, u_{n}$ form mod $v$ a transcendental base of the residue class field $\bar{K}$ of $v$ over $k$, and $n-1$ elements among $x_{1}, \ldots, x_{n}$ form a transcendental base $\bmod v$ of $\bar{K}$. Then if $R \neq 0$,

$$
\begin{aligned}
v(R) & =v\left(\mathfrak{D}_{u_{1} \ldots u_{i l}}\right)-v\left(\mathfrak{D}_{x_{1} \ldots x_{n}}\right)+2\left\{v\left(x_{1}\right)_{\infty}+\ldots+v\left(x_{n}\right)_{\infty}\right\} \\
& -2\left\{v\left(u_{1}\right)_{\infty}+\ldots+v\left(u_{n}\right)_{\infty}\right\} .
\end{aligned}
$$

More generally let $d z_{1} \ldots d z_{s}=\sum_{i_{1}<\ldots<i s} R_{i_{1} \ldots i_{s}} d x_{i_{1}} \ldots d x_{i_{s}}(s \leqq n)$. Suppose further that $n-1$ elements among $\left(z_{1}, \ldots, z_{s}, x_{1}, \ldots, \hat{x}_{i_{1}}, \ldots, \hat{x}_{i_{s}}, \ldots, x_{n}\right)$ form mod $v$ a transcendental base of $\bar{K}$ over $k$. Then if $R_{i_{1} \ldots i_{s}} \neq 0$, 


$$
\begin{aligned}
v\left(R_{i_{1} \ldots i_{s}}\right) & =v\left(\mathfrak{D}_{z_{1} \ldots z_{s} x_{1} \ldots \hat{x}_{i_{2}} \ldots \hat{x}_{i s} \ldots x_{n}}\right)-v\left(\mathfrak{D}_{x_{1} \ldots x_{n}}\right) \\
& +2\left\{v\left(x_{i_{1}}\right)_{\infty}+\ldots+v\left(x_{i_{s}}\right)_{\infty}\right\}-2\left\{v\left(z_{1}\right)_{\infty}+\ldots+v\left(z_{s}\right)_{\infty}\right\} .
\end{aligned}
$$

Proof. We use induction on $n$. The latter part follows immediately from the first part; for, $d z_{1} \ldots d z_{s} d x_{s+1} \ldots d x_{n}=R_{1 \ldots s} d x_{1} \ldots d x_{s} d x_{s+1} \ldots d x_{n}$ (if $\left.i_{1}=1, \ldots, i_{s}=s\right)$.

We assume without loss of generality that $\bar{K} / k\left(\bar{x}_{1}, \ldots, \bar{x}_{n-1}\right)$ and $\bar{K} / k\left(\bar{u}_{1}\right.$, $\ldots, \bar{u}_{n-1}$ ) are algebraic. We put

$$
d u_{1} \ldots d u_{n-1}=A_{1} d x_{2} \ldots d x_{n}+A_{2} d x_{1} d x_{3} \ldots d x_{n}+\ldots+A_{n} d x_{1} \ldots d x_{n-1} \text {. }
$$

I) The case when at least one of $A_{1}, \ldots, A_{n-1}$ is not zero; we assume $A_{1} \neq 0$. Let $k\left(x_{1}\right)^{*}$ be the algebraic closure of $k\left(x_{1}\right)$ in $K$ and consider $K$ over $k\left(x_{1}\right)^{*}$. Then by the induction sssumption we can prove in the same way as Lemma 4 ,

$$
\begin{aligned}
v\left(A_{1}\right) & =v\left(\mathscr{D}_{u_{1} \ldots u_{i-1} x_{1}}\right)-v\left(\mathscr{D}_{x_{1} \ldots x_{n}}\right)+2\left\{v\left(x_{2}\right)_{\infty}+\ldots+v\left(x_{n}\right)\right\}_{\infty} \\
& -2\left\{v\left(u_{1}\right)_{\infty}+\ldots+v\left(u_{n-1}\right)_{\infty}\right\} .
\end{aligned}
$$

Next as $K / k\left(u_{1}, \ldots, u_{n-1}, x_{1}\right)$ is separably algebraic, we can put

$$
d u_{n}=\alpha_{1} d u_{1}+\alpha_{2} d u_{2}+\ldots+\alpha_{n-1} d u_{n-1}+\alpha_{n} d x_{1} .
$$

By Lemma 4 we get

$$
v\left(\alpha_{n}\right)=v\left(\mathfrak{D}_{u_{1} \ldots u_{n-1} u_{n}}\right)-v\left(\mathfrak{D}_{u_{1} \ldots u_{n-1} x_{1}}\right)+2 v\left(x_{1}\right)_{\infty}-2 v\left(u_{n}\right)_{\infty} .
$$

As $R=A_{1} \alpha_{n}$

$$
\begin{aligned}
v(R) & =v\left(A_{1}\right)+v\left(\alpha_{n}\right)=v\left(\mathfrak{D}_{u_{1} \ldots u_{n}}\right)-v\left(\mathfrak{D}_{x_{1} \ldots x_{n}}\right) \\
& +2\left\{v\left(x_{1}\right)_{\infty}+\ldots+v\left(x_{n}\right)_{\infty}\right\}-2\left\{v\left(u_{1}\right)_{\infty}+\ldots+v\left(u_{n}\right)_{\infty}\right\} .
\end{aligned}
$$

II) When $A_{1}=\ldots=A_{n-1}=0$, then $A_{n} \neq 0$, as $R \neq 0$. Put

$$
d u_{1}=\alpha_{1} d x_{1}+\ldots+\alpha_{n} d x_{n}
$$

There exists an element $w$ of $K$ which satisfies the following conditions 1) $K / k\left(x_{1}, \ldots, x_{n-1}, w\right)$ is separably algebraic, 2$) w$ and $n-2$ elements among $x_{1}, \ldots, x_{n-1}$ form mod $v$ a transcendental base of $\bar{K}$ over $k$. For, at first $\bar{u}_{1}$ and $n-2$ elements among $\bar{x}_{1}, \ldots, \bar{x}_{n-1}$ form a transcendental base of $\bar{K}$. We assume that $\bar{u}_{1}, \bar{x}_{2}, \ldots, \bar{x}_{n-1}$ is a transcendental base of $\bar{K}$. As $\bar{K}$ is $(n-1)$ dimensional over $k$, there exists an element $f\left(x_{1}, \ldots, x_{n}\right) \neq 0$ in $k\left[x_{1}, \ldots, x_{n}\right]$ 
such that $v(f)>0$, moreover we can assume that $\partial f / \partial x_{n} \neq-\alpha_{n}$. We put $w=u_{1}$ $+f$. Then, 1) $d w=d u_{1}+d f=\left(\alpha_{1}+\frac{\partial f}{\partial x_{1}}\right) d x_{1}+\ldots+\left(\alpha_{n}+\frac{\partial f}{\partial x_{n}}\right) d x_{n}, \alpha_{n}+\frac{\partial f}{\partial x_{n}}$ $\neq 0$; therefore $K$ is separably algebraic over $k\left(x_{1}, x_{2}, \ldots, x_{n-1}, w\right)$. 2) As $v(f)>0, \bar{w}=\bar{u}_{1}$; therefore $\bar{w}, \bar{x}_{2}, \ldots, \bar{x}_{n-1}$ is a transcendental base of $\bar{K}$.

As $d u_{1} \ldots d u_{n-1}=0 \cdot d x_{2} \ldots d x_{n-1} d w+\ldots+A_{n} d x_{1} \ldots d x_{n-1}$, by considering $K$ over $k(w)^{*}$, we get in the same way as Lemma 4

$$
\begin{aligned}
v\left(A_{n}\right) & =v\left(\mathscr{D}_{u_{1} \ldots u_{n-1} w}\right)-v\left(\mathscr{D}_{w x_{1} \ldots x_{n-1}}\right)+2\left\{v\left(x_{2}\right)_{\infty}+\ldots+v\left(x_{n-1}\right)_{\infty}\right\} \\
& -2\left\{v\left(u_{1}\right)_{\infty}+\ldots+v\left(u_{n-1}\right)_{\infty}\right\} .
\end{aligned}
$$

Since $A_{n} \neq 0$, by Lemma $2, K$ is separably algebraic over $k\left(u_{1}, \ldots, u_{n-1}, w\right)$, and we can put

$$
d u_{n}=\beta_{1} d u_{1}+\ldots+\beta_{n-1} d u_{n-1}+\beta_{n} d w
$$

By Lemma 4

$$
\begin{aligned}
v\left(\beta_{n}\right)= & v\left(\mathfrak{D}_{u_{1} \ldots u_{n}}\right)-v\left(\mathfrak{D}_{u_{1} \ldots u_{n-1} w}\right)+2 v(w)_{\infty}-2 v\left(u_{n}\right)_{\infty} \\
& d w=\gamma_{1} d x_{1}+\ldots+\gamma_{n-1} d x_{n-1}+\gamma_{n} d x_{n} \\
v\left(\gamma_{n}\right)= & v\left(\mathfrak{D}_{w x_{1} \ldots x_{n-1}}\right)-v\left(\mathfrak{D}_{x_{1} \ldots x_{n}}\right)+2 v\left(x_{n}\right)_{\infty}-2 v(w)_{\infty} .
\end{aligned}
$$

As $R=A_{n} \beta_{n} \gamma_{n}$

$$
\begin{aligned}
v(R)= & v\left(A_{n}\right)+v\left(\beta_{n}\right)+v\left(\gamma_{n}\right) \\
= & v\left(\mathfrak{D}_{u_{1} \ldots u_{n}}\right)-v\left(\mathfrak{D}_{x_{1} \ldots x_{n}}\right) \\
& +2\left\{v\left(x_{1}\right)_{\infty}+\ldots+v\left(x_{n}\right)_{\infty}\right\}-2\left\{v\left(u_{1}\right)_{\infty}+\ldots+v\left(u_{n}\right)_{\infty}\right\} .
\end{aligned}
$$

Lemma 5. Let $V^{n}$ be a variety defined over a field $k$ with a generic point $P$ over $k$. Let $W^{n-1}$ be a simple subvariety of $V^{n}$ algebraic over $k$ with a generic point $Q$ over $\vec{k}$. Let $\left(t_{1}, \ldots, t_{n}\right)$ be a set of uniformizing parameters at $Q$ in $k(P)$ and $t_{i}^{\prime}$ be the specialization of $t_{i}$ over $P \rightarrow Q$ with respect to $k$. Then $k\left(x^{\prime}\right)$ is separably algebraic over $k\left(t_{1}^{\prime}, \ldots, t_{n}^{\prime}\right)$.

Proof. From the definition of uniformizing parameters and F-VIII, Prop. $10, Q$ is a proper specialization of multiplicity 1 over $(t) \rightarrow\left(t^{\prime}\right)$ with respect to $k$. Therefore $k\left(x^{\prime}, t^{\prime}\right)=k\left(x^{\prime}\right)$ is separable over $k\left(t^{\prime}\right)$ by F-III, Th. 4 .

Lемма 6. Let $V^{n}$ be a variety defined over a perfect field $k$ with generic point $P$ over $k$. Let $v$ be a valuation of $k(P)$ such that its valuation ring coincides with the specialization ring, in $k(P)$, of a simple subvariety $W^{n-1}$ wnich 
is algebraic over $k$. Then we can choose a set of uniformizing parameters $\left(t_{1}, \ldots, t_{n}\right)$ in $k(P)$ along $W$ such that $v\left(t_{1}\right)=$ minimum of the set consisting of $v(\alpha), \alpha \in k(P), v(\alpha)>0$.

Proof. There is a point $A=\left(x^{\prime \prime}\right)$ algebraic over $k$ such that $A$ is a simple point both on $V^{n}$ and on $W^{n-1}$. Let $\mathfrak{p}$ be the prime ideal in the specialization ring $R$ of $\left(x^{\prime \prime}\right)$ in $k(P)$ which is determined by $Q$. Then since $\left(x^{\prime \prime}\right)$ is simple on $W$ the ring $R / \mathfrak{p}$ is a regular local ring. If $\left(t_{2}, \ldots, t_{n}\right)$ is a set of elements in $R$ such that they form mod $p$ a regular system of parameters of $R / p$, then there is an element $t_{1}$ in $R$ which is a generator of $\mathfrak{p}$ and $\left(t_{1}, \ldots, t_{n}\right)$ is a regular system of parameters of $R$ by Chevalley [1], Prop. 9. Since $k$ is perfect, $\left(t_{1}, \ldots, t_{n}\right)$ is a set of uniformizing parameters of $V$ at $A$ in $k(x)$. Therefore $\left(t_{1}, \ldots, t_{n}\right)$ is a set of uniformizing parameters of $V$ at $Q$ in $k(P)$ and $v\left(t_{1}\right)=$ minimum.

THEOREM 2.4) Let $K$ be a regular $n$-dimensional extension over a perfect field $k$, let $v$ be an $(n-1)$-dimensional valuation of $K$ and let $V^{n}$ be a model of $K$ such that the center of $v$ is a simple subvariety $W^{n-1}$ and $\left(t_{1}, \ldots, t_{n}\right)$ a set of uniformizing parameters of $W^{5)}$ Let $z_{1}, \ldots, z_{n}$ be elements in $K$ such that the residue-class field of $v$ is algebraic over $k\left(\bar{z}_{1}, \ldots, \bar{z}_{n}\right)$, where $\bar{z}_{i}$ is the residue class which contains $z_{i}$ (when $v\left(z_{i}\right)<0$ we consider here $\bar{z}_{i}=0$ ). Then if

$$
\begin{gathered}
d z_{1} \ldots d z_{n}=w d t_{1} \ldots d t_{n} \quad \text { and } \quad w \neq 0, \\
v(w)=v\left(\mathfrak{D}_{z_{1} \ldots z_{n}}\right)-2\left\{v\left(z_{1}\right)_{\infty}+\ldots+v\left(z_{n}\right)_{\infty}\right\} .
\end{gathered}
$$

Proof. For the set of uniformizing parameters $\left(t_{1}, \ldots, t_{n}\right)$ which was chosen in Lemma $6, v\left(\mathfrak{D}_{t_{1} \ldots t_{n}}\right)=0$ since $v\left(t_{1}\right)=$ minimum and the residue class field of $v$ is separable over $k\left(\bar{t}_{1}, \ldots, \bar{t}_{n}\right)$. As $v(w)$ is independent of the choice of the uniformizing parameters, we get the theorem.

Corollary. Let $k(x)$ be a separably algebraic extension of $k(y)$ and let $v^{*}$ be a valuation in $k(x)$ which induces on $k(y)$ an $(n-1)$-dimensional valuation $v, n$ being the dimension of $k(y)$ over $k$. Let $\left(t_{1}^{*}, \ldots, t_{n}^{*}\right)$ be a set of uniformizing parameters of $v^{*}$ in $k(x)$ and let $\left(t_{1}, \ldots t_{n}\right)$ be a set of uniformizing parameters of $v$ in $k(y)$ in the sense described in the above theorem, and

4) See H. W. E. Jung [5].

5) Since $k$ is perfect, such a variety always exists. 


$$
d t_{1} \ldots d t_{n}=w d t_{1}^{*} \ldots d t_{n}^{*} \quad \text { in } k(x)
$$

Then $v^{*}(w)=v^{*}\left(\mathfrak{D}_{k(x) / k(y)}\right)$.

Proof. By Theorem $2 v^{*}(w)=v^{*}\left(\mathfrak{D}_{t_{1} \ldots t_{n}}\right)$, but $v^{*}\left(\mathfrak{D}_{t_{1} \ldots t_{n}}\right)=v^{*}\left(\mathfrak{D}_{k(x) / k(y)}\right)$, because the $v$-contribution of the different of $k(y)$ with respect to $k\left(t_{1}, \ldots, t_{n}\right)$ is zero as in the proof of Theorem 2.

$\S 2$. Let $V^{n}$ be a projective variety in the projective $n+1$ space $L^{n+1}$. Let $k$ be a field of definition of $V^{n}$, and let $V^{* n}$ be a derived normal variety of $V$ with respect to $k$, such that $V^{*}$ has no singular subvariety of dimension $n-1$. $^{6}$ Then $V^{*}$ is also derived normal variety of $V$ with respect to any field $k^{\prime}$ containing $k$. Let $M$ be a generic point of $V$ over $k$, and let $M^{*}$ be the corresponding generic point of $V^{*}$ over $k$. Let $W^{* n-1}$ be an $(n-1)$-dimensional subvariety of $V^{*}$ and let $W$ be the corresponding variety in $V$. Let $\mathfrak{D}_{W}$ be the specialization ring of $W$ in $k(M)$, and $\overline{\mathfrak{D}}_{W}$ the integral closure of $\mathfrak{o}_{W}$ in $k(M)$ $=k\left(M^{*}\right)$. Let $\left(_{W}\right.$ be the conductor of $\overline{\mathfrak{D}}_{W}$ with respect to $\mathfrak{o}_{W}$, and put $c_{W^{*}}$ $=\min _{u \in \mathbb{C}_{W}}\left\{v_{W^{*}}(u)\right\}$, where $v_{W^{*}}$ means the valuation of $k\left(M^{*}\right)$ with respect to $W^{*}$. We define the subadjoint divisor $C$ of $V$ by $C=\sum c_{W^{*}} W^{*}{ }^{7}$ ) $\quad$ Here $c_{W^{*}} \neq 0$ if and only if $W$ is a singular subvariety of $V$.

Let $W_{0}, V_{0}$ and $V_{0}^{*}$ be representatives of $W, V$ and $V^{*}$ respectively and let $M_{0}=(x)$ and $M_{0}^{*}=(y)$ be the corresponding generic points of $V_{0}$ and $V_{0}^{*}$ over $k$ respectively. Let $z_{1}, \ldots, z_{m}$ be a base of the ring $k[y]$ with respect to $k[x]$. Then $\left(z_{1}, \ldots, z_{m}\right)$ is also a base of $\bar{D}_{W}$ with respect to $\mathfrak{D}_{W}$. From this we see that if $\mathfrak{s}$ is the conductor of $k[y]$ with respect to $k[x]$ then $\mathfrak{\mho}_{W}=\mathfrak{s} \cdot \mathfrak{o}_{W}$. For if $u \in \mathfrak{S}_{W}, u z_{i} \in \mathfrak{o}_{W}(i=1, \ldots, m), u z_{i}=\frac{g_{i}(x)}{h_{i}(x)}, g_{i}(x), h_{i}(x) \in k[x], v_{W^{*}}\left(h_{i}(x)\right)$ $\neq 0$; therefore $u \in \mathbb{S} \cdot \mathfrak{D}_{W}$ since $u \prod_{i=1}^{m} h_{i}(x) \in \mathbb{\mho}$. Conversely we get obviously

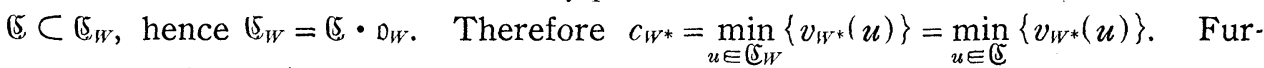
ther since $V^{*}$ is derived normal variety of $V$ with respect to $k^{\prime}$, if $\left(z_{1}, \ldots, z_{m}\right)$ is a base of $k[y]$ with resp. to $k[x]$ it is also a base of $k^{\prime}[y]$ with resp. to $k^{\prime}[x]$, therefore the conductor $\mathfrak{S}^{\prime}$ of $k^{\prime}[y]$ with respect to $k^{\prime}[x]$ is equal to (5. $k^{\prime}[x]$. Therefore we can see that $C$ depends on the variety $V^{*}$, but it does not depend on the choice of the reference field $k$ and the generic point over it.

6) In the following we always assume that the derived variety of $V$ by normalization with reference to $k$ is normal and call derived normal variety.

7) See D. Gorenstein [2]. 
Moreover $C$ is clearly determined uniquely up to biregular birational correspondences between derived normal varieties.

Let $\Phi\left(X_{0}, \ldots, X_{n+1}\right)$ be a homogeneous form in $k\left[X_{0}, \ldots, X_{n+1}\right]$. Let $W^{* n-1}$ be an $(n-1)$-dimensional subvariety in $V^{*}$ and $W$ the corresponding subvariety in $V$. We take one of the representatives, say $W_{0}$, of $W$. Let $M_{0}$ $=\left(1, x_{1}, \ldots, x_{n+1}\right)$ be a generic point of $V_{0}$ over $k$ and $M^{*}$ be the correspond ing generic point of $V^{*}$ over $k$. Let $\Phi_{0}$ be the function on $V^{*}$ defined by $\Phi_{0}\left(M^{*}\right)=\Phi\left(1, x_{1}, \ldots, x_{n+1}\right)$ over $k$. Then it is easily seen that $v_{W^{*}}\left(\Phi_{0}\right)$ is independent of the choice of the representatives of $W$ and the field $k$ and the generic point $M^{*}$ of $V^{*}$ over $k$. We denote it by $v_{W^{*}}(\Phi, V)$. Obviously $v_{W^{*}}(\Phi, V)=0$ but a finite number of $W^{*}$; we denote the divisor $\sum_{W^{*}} v_{W^{*}}(\Phi, V) \cdot W^{*}$ by $(\Phi, V)$. If $v_{W^{*}}(\Phi, V) \geqslant v_{W^{*}}(C)$ for all $W^{*}$, then we call that $\Phi\left(X_{n}, \ldots, X_{n+1}\right)$ is a subadjoint form of $V$ or call the hypersurface $\Phi=0$ a subadjoint hypersurface. Let $\phi\left(X_{1}, \ldots, X_{n+1}\right)$ be a polynomial in $k\left[X_{1}, \ldots, X_{n+1}\right]$ and let $\phi$ be the function on $V^{*}$ defined by $\phi\left(M^{*}\right)=\phi\left(x_{1}, \ldots, x_{n+1}\right)$. If $v_{W^{*}}(\phi) \geqslant v_{W^{*}}(C)$ for all $W^{*}$ such that $W^{*}$ has the representative in $V_{0}^{*}$, then we say that $\phi$ is a subadjoint polynomial of $V_{0}$. If the degree $s$ of $\phi$ is $\leqq m$, and $\mathscr{D}\left(X_{0}, \ldots, X_{n+1}\right)$ $=X_{0}^{m} \cdot \phi\left(\frac{X_{1}}{X_{0}}, \ldots, \frac{X_{n+1}}{X_{0}}\right)$ is a subadjoint form of $V$ we say that $\phi\left(X_{1}, \ldots\right.$, $\left.X_{n+1}\right)=0$ defines a subadjoint hypersurface of degree $m$ of $V$, or briefly $\phi\left(X_{1}\right.$, $\left.\ldots, X_{n+1}\right)$ is a subadjoint hypersurface of degree $m$ of $V$. The notion of subadjointness is independent of $k$ and also of $V^{*}$.

Let $\omega$ be a differential form on $V$ of degree $r$ defined by $\omega(M)$ $=\sum_{i_{1}<\ldots<i_{r}} a_{i_{1} \ldots i_{r}} d x_{i_{1}} \ldots d x_{i_{r}}$ over $k$, where $\sum a_{i_{1} \ldots i_{r}} d x_{i_{1}} \ldots d x_{i_{r}}$ is a differential form belonging to the extension $k(M)$ of $k$. In this case for simplicity we also use the notation $\omega=\sum_{i_{1}<\ldots<i_{r}} a_{i_{1} \ldots i_{r}} d x_{i_{1}} \ldots d x_{i_{r}}$. Further by the birational correspondence between $V^{*}$ and $V$, we can define the transformed differential form for $\omega$ on $V^{*}$, using the same notation $\omega$.

Let $\omega$ be a differential form defined over $k$ on $V^{*}$ and let $\left(t_{1}, \ldots t_{n}\right)$ be a set of uniformizing parameters of a subvariety $W^{* n-1}$ in $k(M)$. If $\omega=\sum a_{i_{1} \ldots i_{r}} d t_{i_{1}} \ldots d t_{i_{r}}$, then we put $\min . v_{W^{*}}\left(a_{i_{1} \ldots i_{r}}\right)=v_{W^{*}}(\omega)$; this is independent of the choice of the uniformizing parameters and also of the defining field $k^{8)}$

8) See Y. Nakai [7]. 
Lemma 7. Let $V^{n}$ be an affine variety in $S^{n+1}$ defined over $k$ by $F\left(X_{1}, \ldots\right.$, $\left.X_{n+1}\right)=0$, with a generic point $M=\left(x_{1}, \ldots, x_{n+1}\right)$ over $k$. We assume that $x_{n+1}$ is separably algebraic over $k\left(x_{1}, \ldots, x_{n}\right)$. Let $\omega=\frac{\phi\left(x_{1}, \ldots, x_{n+1}\right)}{F_{n+1}^{\prime}\left(x_{1}, \ldots, x_{n+1}\right)} d x_{1}$ $\ldots d x_{n}$, and let $W^{* n-1}$ be a subvariety of the variety $V^{*}$ derived from $V$ by normalization with reference to $k$. Then $v_{W^{*}}(\omega)=v_{W^{*}}(\phi)-v_{W^{*}}(C)$.

Proof. (1) First we assume that the degree of $F\left(X_{1}, \ldots, X_{n+1}\right)$ on $X_{n+1}$ is $m, m$ being the degree of $F\left(X_{1}, \ldots, X_{n+1}\right)$. Let $W^{n-1}$ be the corresponding variety to $W^{*}$ in $V$ and let $\left(x^{\prime}\right)$ be a generic point of $W$ over $k^{\prime}(\supseteqq k)$. Since $F\left(x_{1}^{\prime}, \ldots, x_{n}^{\prime}, x_{n+1}^{\prime}\right)=0, x_{n+1}^{\prime}$ is algebraic over $k^{\prime}\left(x_{1}^{\prime}, \ldots, x_{n}^{\prime}\right)$, and therefore $\operatorname{dim}_{k^{\prime}}\left(x_{1}^{\prime}, \ldots, x_{n}^{\prime}\right)=n-1$. Suppose $x_{1}^{\prime}, \ldots, x_{n-1}^{\prime}$ be algebraically independent over $k^{\prime}$. Then for any element $z \neq 0$ in $k\left(x_{1}, \ldots, x_{n-1}\right)=k_{1}, v_{W^{*}}(z)=0$, and therefore we can consider $v_{W^{*}}$ as a valuation of $k_{1}\left(x_{n}, x_{n+1}\right)$ over $k_{1}$. Let $\mathfrak{o}$ be the integral closure of $k_{1}\left[x_{n}\right]$ in $K=k\left(x_{1}, \ldots, x_{n}, x_{n+1}\right)$. Then $\mathfrak{D}$ is also the integral closure of $k_{1}\left[x_{n}, x_{n+1}\right]$. Let $\mathrm{c}$ be the conductor of $\mathfrak{D}$ with respect to $k_{1}\left[x_{n}, x_{n+1}\right]$ and let $\delta$ be the different of $\mathfrak{o}$ with respect to $k_{1}\left[x_{n}\right]$. Then we get

$$
F_{n+1}^{\prime}\left(x_{1}, \ldots, x_{n}, x_{n+1}\right) \cdot \mathrm{D}=\mathrm{CD}^{9)}
$$

Further, clearly we get $v_{W^{*}}(C)=v_{W^{*}}\left(\mathfrak{C}_{W}\right)=v_{W^{*}}(\mathrm{C})$, and moreover we get $v_{W^{*}}(\delta)$ $=v_{W^{*}}\left(\mathfrak{D}_{x_{1} \ldots x_{n}}\right)$. As we can assume without loss of generality that $k$ is perfect, we get

Therefore

$$
v_{W^{*}}\left(d x_{1} \ldots d x_{n}\right)=v_{W^{*}}\left(\mathfrak{D}_{x_{1} \ldots x_{n}}\right)
$$

$$
\begin{aligned}
v_{W^{*}}(\omega) & =v_{W^{*}}(\phi)+v_{W^{*}}\left(\mathfrak{D}_{x_{1} \ldots x_{n}}\right)-v_{W^{*}}\left(F_{n+1}^{\prime}\left(x_{1}, \ldots, x_{n+1}\right)\right) \\
& =v_{W^{*}}(\phi)+v_{W^{*}}\left(\mathfrak{D}_{x_{1} \ldots x_{n}}\right)-\left[v_{W^{*}}(C)+v_{W^{*}}\left(\mathfrak{D}_{x_{1} \ldots x_{n}}\right)\right] \\
& =v_{W^{*}}(\phi)-v_{W^{*}}(C)
\end{aligned}
$$

(2) For the general case we make a linear transformation $\bar{x}_{i}=\sum_{j=1}^{n+1} a_{i j} x_{j}$, where $a_{i j}$ are in $k$ and $\left|a_{i j}\right| \neq 0$.

$$
\begin{gathered}
d \bar{x}_{i}=\sum_{j=1}^{n+1} a_{i j} d x_{j}=\sum_{j=1}^{n}\left(a_{i j}-\frac{F_{j}^{\prime}}{F_{n+1}^{\prime}} a_{i, n+1}\right) d x_{j} \\
d \bar{x}_{1} \ldots d \bar{x}_{n}=\left|a_{i j}-\frac{F_{j}^{\prime}}{F_{n+1}^{\prime}} a_{i, n+1}\right|_{i, j=1, \ldots, n} d x_{1} \ldots d x_{n} \\
x_{i}=\sum_{j=1}^{n+1} \alpha_{i j} \bar{x}_{j} \quad(i=1, \ldots, n+1), \quad\left(\alpha_{i j}\right)=\left(a_{i j}\right)^{-1} .
\end{gathered}
$$

9) E. Hecke [3]. 
Let $\bar{F}\left(\bar{X}_{1}, \ldots, \bar{X}_{n+1}\right)$ be the irreducible polynomial in $k\left[\bar{X}_{1}, \ldots, \bar{X}_{n+1}\right]$ such that $\bar{F}\left(\bar{x}_{1}, \ldots, \bar{x}_{n+1}\right)=0$. Then $\bar{F}\left(\bar{X}_{1}, \ldots, \bar{X}_{n+1}\right)=F\left(\sum_{j=1}^{n+1} \alpha_{1 j} \bar{X}_{j}, \ldots, \sum_{j=1}^{n+1} \alpha_{n+1, j} \bar{X}_{j}\right)$, $\bar{F}_{x_{n+1}}^{\prime}=F_{1}^{\prime} \cdot \alpha_{1, n+1}+F_{2}^{\prime} \cdot \alpha_{2, n+1}+\ldots+F_{n+1}^{\prime} \alpha_{n+1, n+1}$,

$$
\frac{\bar{F}_{x_{n+1}}^{\prime}}{F_{n+1}^{\prime}}=\frac{\left|a_{j i}-\frac{F_{j}^{\prime}}{F_{n+1}^{\prime}} a_{i n+1}\right|_{i, j=1, \ldots, n}}{\left|a_{i j}\right|_{i, j=1, \ldots, n+1}}
$$

Therefore

$$
\begin{gathered}
\frac{1}{F_{n+1}^{\prime}} d x_{1} \ldots d x_{n}=\frac{\left|a_{i j}\right|^{-1}}{\bar{F}_{\bar{x}_{n+1}}^{\prime}} d \bar{x}_{1} \ldots d \bar{x}_{n} \\
\omega=\frac{\phi\left(x_{1}, \ldots, x_{n+1}\right)}{F_{n+1}^{\prime}\left(x_{1}, \ldots, x_{n+1}\right)} d x_{1} \ldots d x_{n}=\frac{\phi\left(x_{1} \ldots x_{n+1}\right)\left|a_{i j}\right|^{-1}}{\bar{F}_{x_{n+1}}^{\prime}\left(\bar{x}_{1}, \ldots, \bar{x}_{n+1}\right)} d \bar{x}_{1} \ldots d \bar{x}_{n}
\end{gathered}
$$

Since we can assume that $k$ is an infinite field, we can select $a_{i j}$ such that $\bar{F}$ is of order $m$ on $\bar{X}_{n+1}$. Therefore we have

$$
v_{W^{*}}(\omega)=v_{W^{*}}(\phi)-v_{W^{*}}(C) .
$$

TheOREM 3. Let $V^{n}$ be an affine variety in $S^{n+1}$ defined over $k$ by $F\left(X_{1}\right.$, $\left.\ldots, X_{n+1}\right)=0$ with a generic point $M=\left(x_{1}, \ldots, x_{n+1}\right)$ over $k$. We assume $x_{n+1}$ is separably algebraic over $k\left(x_{1}, \ldots, x_{n}\right)$. Then a differential form $\omega=\frac{A}{F_{n+1}^{\prime}\left(x_{1}, \ldots, x_{n+1}\right)} d x_{1} \ldots d x_{n}$ is finite at every simple point of the derived normal variety $V^{*}$ if and only if $A=\phi\left(x_{1}, \ldots, x_{n+1}\right)$, where $\phi\left(X_{1}, \ldots, X_{n+1}\right)$ is a subadjoint polynomial for $V$.

Proof. If $A=\phi\left(x_{1}, \ldots, x_{n+1}\right), \phi\left(X_{1}, \ldots, X_{n+1}\right)$ is a subadjoint polynomial for $V$, then $v_{W^{*}}\left(\phi\left(x_{1}, \ldots, x_{n+1}\right)\right) \geqslant v_{W^{*}}(C)$ for every subvariety $W^{* n-1}$ of $V^{*}$. By the preceding lemma $v_{W^{*}}(\omega)=v_{W^{*}}(\phi)-v_{W^{*}}(C) \geq 0$. Let $P$ be any simple point of $V^{*}$ and let $\left(t_{1}, \ldots, t_{n}\right)$ be a set of uniformizing parameters at $P$ in $k(M)$ and $\omega=B d t_{1} \ldots d t_{n}$. Since $\left(t_{1}, \ldots, t_{n}\right)$ is also a set of uniformizing parameters along every $W^{* n-1}$ which contains $P$. Therefore $v_{W^{*}}(B) \gtrsim 0$ for every $W^{*}$ which contains $P$. It follows by F-VII, Th. 1 that $B$ must belong to the specialization ring of $P$; this shows that $\omega$ is finite at every simple point.

Conversely if $\omega$ is finite at every simple point, then $v_{W^{*}}(\phi) \geqslant v_{W^{*}}(C)$ for every $W^{* n-1}$ of $V^{*}$. Let $\mathrm{D}_{W}$ be the specialization ring of a subvariety. $W^{n-1}$ of $V$ in $k(M)$, and let $\overline{0}_{W}$ be the integral closure of $\mathrm{o}_{W}$ in $k(M)$. Let $W_{i}^{* n-1}$ $(i=1, \ldots, s)$ be all the subvarieties of $V^{*}$ which correspond to $W$. Then an element $z$ in $k(M)$ is finite at $W$ (which means $z$ is finite at a generic point 
of $W$ over a field of definition $K(\supseteqq k)$ of $W$ ), if and only if $z$ is finite at every $W_{i}^{*}(i=1, \ldots, s)$. Therefore $\overline{\mathfrak{D}}_{W}$ is the intersection of the specialization rings of $W_{i}^{*}$ 's. It follows that $\overline{\mathfrak{D}}_{W}$ is a principal ideal ring. Hence we have an element $c$ in $\mathbb{E}_{W}$ such that $v_{W_{i}}(C)=\min _{u \in \mathfrak{C}_{W}}\left\{v_{W_{i}}(u)\right\}=v_{W i^{*}}(c)$, therefore we conclude that $\phi / c \in \overline{\mathfrak{D}}_{W}, \phi \in \mathfrak{\complement}_{W}$.

If $z \in \bigcap_{W^{n-1} C V} \mathfrak{D}_{W}$, then $z$ belongs to $k\left[x_{1}, \ldots, x_{n+1}\right]$. To prove this, we may assume that $F\left(X_{1}, \ldots, X_{n+1}\right)$ is of degree $m$ and it contains a term of $X_{n+1}^{m}$; under this assumption $\left(x_{1}, \ldots, x_{n}\right)$ is a set of independent elements over $k$. Since $z \in \in_{W^{n-1} C_{V}} \hat{O}_{W V}$, it belongs to the specialization ring, in $k\left(x_{1}, \ldots\right.$, $\left.x_{n-1}\right)\left(x_{n}, x_{n+1}\right)$, of any specialization $\left(x_{n}^{\prime}, x_{n+1}^{\prime}\right)$ of $\left(x_{n}, x_{n+1}\right)$ with reference to $k\left(x_{1}, \ldots, x_{n-1}\right)$, therefore we can see that $z$ belongs to $k\left(x_{1}, \ldots, x_{n-1}\right)$ $\left[x_{n}, x_{n+1}\right]$; hence

$$
z=\frac{g\left(x_{1}, \ldots, x_{n+1}\right)}{h\left(x_{1}, \ldots, x_{n-1}\right)}
$$

where $h\left(X_{1}, \ldots, X_{n-1}\right) \in k\left[X_{1}, \ldots, X_{n-1}\right]$ and $g\left(X_{1}, \ldots, X_{n+1}\right) \in k\left[X_{1}\right.$, $\left.\ldots, X_{n+1}\right]$, moreover since $F\left(X_{1}, \ldots, X_{n+1}\right)$ is of degree $m$ on $X_{n+1}$, we may assume that $g\left(X_{1}, \ldots, X_{n+1}\right)$ is of degree $<m$ on $X_{n+1}$. Similarly $z$ belongs to $k\left(x_{1}, \ldots, x_{n-2}, x_{n}\right)\left[x_{n-1}, x_{n+1}\right]$, and

$$
z=\frac{g_{1}\left(x_{1}, \ldots, x_{n+1}\right)}{h_{1}\left(x_{1}, \ldots, x_{n-2}, x_{n}\right)}
$$

where $h_{1}\left(X_{1}, \ldots, X_{n-2}, X_{n}\right) \in k\left[X_{1}, \ldots, X_{n-2}, X_{n}\right], g_{1}\left(X_{1}, \ldots, X_{n+1}\right) \in k\left[X_{1}\right.$, $\left.\ldots, X_{n+1}\right]$ and $g_{1}\left(X_{1}, \ldots, X_{n+1}\right)$ is of degree $<m$ on $X_{n+1}$. Therefore

$$
\begin{aligned}
g_{1}\left(X_{1}, \ldots, X_{n+1}\right) & \cdot h\left(X_{1}, \ldots, X_{n-1}\right) \\
& -g\left(X_{1}, \ldots, X_{n+1}\right) \cdot h\left(X_{1}, \ldots, X_{n-2}, X_{n}\right)
\end{aligned}
$$

is divisible by $F\left(X_{1}, \ldots, X_{n+1}\right)$, but since its degree on $X_{n+1}$ is $<m$

$$
\begin{aligned}
g_{1}\left(X_{1}, \ldots, X_{n+1}\right) & h\left(X_{1}, \ldots, X_{n-1}\right) \\
& =g\left(X_{1}, \ldots, X_{n+1}\right) h_{1}\left(X_{1}, \ldots, X_{n-2}, X_{n}\right) .
\end{aligned}
$$

Now if $H\left(X_{1}, \ldots, X_{n-1}\right)$ is a power of an irreducible polynomial, which has a term containing $X_{n-1}$, and devides $h\left(X_{1}, \ldots, X_{n-1}\right)$, then $H\left(X_{1}, \ldots, X_{n-1}\right)$ must devide $g\left(X_{1}, \ldots, X_{n+1}\right)$. Therefore we get

$$
z=\frac{g^{\prime}\left(x_{1}, \ldots, x_{n+1}\right)}{h^{\prime}\left(x_{1}, \ldots, x_{n-2}\right)}
$$


Since $z$ also belongs to $k\left(x_{1}, \ldots, x_{n-5}, x_{n-1}, x_{n}\right)\left[x_{n-2}, x_{n}\right]$, we get

$$
\begin{aligned}
g^{\prime}\left(X_{1}, \ldots, X_{n+1}\right) h_{2}\left(X_{1}, \ldots, X_{n-3}, X_{n-1}, X_{n}\right) \\
\\
=h^{\prime}\left(X_{1}, \ldots, X_{n-2}\right) g_{2}\left(X_{1}, \ldots, X_{n+1}\right)
\end{aligned}
$$

and similarly as above we can see

$$
z=\frac{g^{\prime \prime}\left(x_{1}, \ldots, x_{n+1}\right)}{h^{\prime \prime}\left(x_{1}, \ldots, x_{n-3}\right)}
$$

Continuing this we get $z \in k\left[x_{1}, \ldots x_{n+1}\right]$.

Let $(5$ be the conductor of the ring $\bar{D}$ which consists of the elements which are integral over $k\left[x_{1}, \ldots, x_{n+1}\right]$, with respect to $k\left[x_{1}, \ldots, x_{n+1}\right]$. Since $\phi \in \aleph_{W}, \phi \bar{D} \subset \mathbb{D}_{W}$ for every $W^{n-1}$, therefore $\phi \overline{0} \leqq k\left[x_{1}, \ldots, x_{n+1}\right]$ which shows that $\phi \in \mathbb{C}$; in particular $\phi \in k\left[x_{1}, \ldots, x_{n+1}\right]$. Therefore $\phi$ is a subadjoint polynomial of $V$.

THEOREM 4. Let $V^{n}$ be a projective variety in the projective $n+1$ space $L^{n+1}$, and $\omega$ a differential form on $V$ of degree $n$. Suppose $V$ have a representative $V_{0}$ defined over $k$ by $F\left(X_{1}, \ldots, X_{n+1}\right)=0$ with a generic point $M_{0}$ $=\left(1, x_{1}, \ldots, x_{n+1}\right)$ over $k$. Suppose $x_{n+1}$ be separably algebraic over $k\left(x_{1}, \ldots\right.$, $\left.x_{n+1}\right)$ and let $\omega=\frac{A}{F_{n+1}^{\prime}\left(x_{1}, \ldots, x_{n+1}\right)} d x_{1} \ldots d x_{n} . \quad$ Then, $\omega$ is finite at every simple point of the derived normal variety $V^{*}$ of $V$ if and only if $A=\phi\left(x_{1}\right.$, $\left.\ldots, x_{n+1}\right)$, where $\phi\left(X_{1}, \ldots, X_{n+1}\right)=0$ is a subadjoint hypersurface of degree $m-(n+2)$ of $V$ in $L^{n+1}, m$ being the degree of $F\left(X_{1}, \ldots, X_{n+1}\right)$.

Proof. Let $\omega=\frac{A}{F_{n+1}^{\prime}\left(x_{1}, \ldots, x_{n+1}\right)} d x_{1} \ldots d x_{n}$ be finite at every simple point of $V^{*}$. Then $\omega$ must be finite at every simple point of $V_{0}^{*}$. Therefore by the preceding theorem $A=\phi(x)$, where $\phi(X)$ is a subadjoint polynomial for $V_{0}$. Let $V_{i}(i \neq n+1)$, say $i=1$, be another representative of $V . M_{1}=\left(\bar{x}_{1}\right.$, $\left.1, \bar{x}_{2}, \ldots, \bar{x}_{n+1}\right)$ is a generic point of $V_{1}$ over $k$, where $\bar{x}_{1}=\frac{1}{x_{1}}, \bar{x}_{2}=\frac{x_{2}}{x_{1}}, \ldots$, $\bar{x}_{n+1}=\frac{x_{n+1}}{x_{1}}$. Let $F\left(X_{1}, \ldots, X_{n+1}\right)=\bar{F}\left(\bar{X}_{1}, \ldots, \bar{X}_{n+1}\right) / \bar{X}_{1}^{m}$ where $\bar{X}_{1}=\frac{1}{X_{1}}$, $\bar{X}_{2}=\frac{X_{2}}{X_{1}}, \ldots, \bar{X}_{n+1}=\frac{X_{n+1}}{X_{1}}$. Then $\bar{F}\left(\bar{X}_{1}, \ldots, \bar{X}_{n+1}\right)=0$ is equation for $V_{1}$ over $k$.

$$
\begin{aligned}
d x_{1} \ldots d x_{n} & =-x_{1}^{-2} d \bar{x}_{1} \cdot \bar{x}_{1}^{-1} d \bar{x}_{2} \ldots \bar{x}_{1}^{-1} d \bar{x}_{n} \\
& =-\bar{x}_{1}^{-(n+1)} d \bar{x}_{1} d \bar{x}_{2} \ldots d \bar{x}_{n}
\end{aligned}
$$




$$
\begin{aligned}
\omega & =\frac{\phi_{*}(x)}{F_{n+1}^{\prime}\left(x_{1}, \ldots, x_{n+1}\right)} d x_{1} \ldots d x_{n} \\
& =-\frac{\phi\left(x_{1}, \ldots, x_{n+1}\right) \bar{x}_{1}^{m-(n+2)}}{\bar{F}_{x_{n+1}}^{\prime}\left(\bar{X}_{1}, \ldots, \bar{x}_{n+1}\right)} d \bar{x}_{1} \ldots d \bar{x}_{n}
\end{aligned}
$$

Therefore $\phi(x) \bar{x}_{1}^{m-(n+2)}$ is equal to a polynomial of $\bar{x}_{1}, \ldots, \bar{x}_{n+1}$. Let $\widetilde{x}_{1}=\frac{x_{1}}{x_{n+1}}, \tilde{x}_{2}=\frac{x_{2}}{x_{n+1}}, \ldots, \widetilde{x}_{n}=\frac{x_{n}}{x_{n+1}}, \tilde{x}_{n+1}=\frac{1}{x_{n+1}}$, and let $\widetilde{F}\left(\widetilde{X}_{1}, \ldots, \widetilde{X}_{n+1}\right)$ be equation for $V_{n+1}$. In the case, where one of $F_{1}^{\prime}(x), \ldots, F_{n}^{\prime}(x)$, say $F_{1}^{\prime}(x)$, is not zero, we have $\frac{1}{F_{n+1}^{\prime}(x)} d x_{1} \ldots d x_{n}=-\frac{1}{F_{1}^{\prime}(x)} d x_{n+1} d x_{2} \ldots d x_{n}$. Therefore we get in the same way as above,

$$
\begin{gathered}
\omega=\frac{\phi(x) \widetilde{x}_{n+1}^{m-(n+2)}}{\widetilde{F} \widetilde{x}_{1}(\widetilde{x})} d \widetilde{x}_{n+1} d \widetilde{x}_{2} \ldots d \widetilde{x}_{n} \\
\left(=-\frac{\phi(x) \widetilde{x}_{n+1}^{m-(n+2)}}{\widetilde{F}_{\widetilde{x}_{n+1}}^{\prime}(\widetilde{x})} d \widetilde{x}_{1} \ldots d \widetilde{x}_{n} \text { if further } \widetilde{F}_{\widetilde{x}_{n+1}}^{\prime}(\widetilde{x}) \neq 0\right)
\end{gathered}
$$

Lf $F_{1}^{\prime}(x)=\ldots=F_{n}^{\prime}(x)=0$, then $d x_{n+1}=0$.

$$
\begin{aligned}
& F_{n+1}^{\prime}(x)=-\widetilde{F}_{x_{n+1}}^{\prime}(\widetilde{x}) / \widetilde{x}_{n+1}^{m-2} \\
& d x_{1}=d\left(x_{n+1} \widetilde{x}_{1}\right)=x_{n+1} d \widetilde{x}_{1}=\widetilde{x}_{n+1}^{-1} d \widetilde{x}_{1}
\end{aligned}
$$

Therefore

$$
\omega=\frac{\phi(x)}{F_{n+1}^{\prime}(x)} d x_{1} \ldots d x_{n}=\frac{\phi(x) \widetilde{x}_{n+1}^{m-(n+2)}}{\widetilde{F}^{\prime}(\widetilde{x})} d \widetilde{x}_{1+1}^{\prime} \ldots d \widetilde{x}_{n}
$$

Hence $\phi(x) \widetilde{x}_{n+1}^{m-(n+2)}$ is equal to a polynomial of $\widetilde{x}_{1}, \ldots, \widetilde{x}_{n+1}$. It follows that $A=\phi\left(x_{1}, \ldots, x_{n+1}\right)$, where $\phi\left(X_{1}, \ldots, X_{n+1}\right)$ is a polynomial of degree $h \leqq m$ $-(n+2) ;$ and $\Phi\left(X_{0}, \ldots, X_{n+1}\right)=\phi\left(\frac{X_{1}}{X_{0}}, \ldots, \frac{X_{n+1}}{X_{0}}\right) X_{0}^{m-(n+2)}$ is subadjoint form of $V$. Conversely if $A=\phi(x)$, where $\phi(X)=0$ is a subadjoint hypersurface of degree $m-(n+2)$, then clearly $\omega=\frac{A}{F_{n+1}^{\prime}(x)} d x_{1} \ldots d x_{n}$ is finite at every simple point of $V^{*}$.

Corollary 1. Let $V^{n}$ be a subvariety of $L^{n+1}$, such that the derived normal variety $V^{*}$ of $V$ is a variety without singular point. Then a differential form

$$
\omega=\frac{A}{F_{n+1}^{\prime}\left(x_{1}, \ldots, x_{n+1}\right)} d x_{1} \ldots d x_{n}
$$

on $V$ is a differential form of the first kind if and only if $A=\phi\left(x_{1}, \ldots, x_{n+1}\right)$, where $\phi\left(X_{1}, \ldots, X_{n+1}\right)=0$ is a subadjoint hypersurface of degree $m-(n+2)$ of $V$. 
Corollary 2. Let $V^{n} \subset L^{n+1}$. If every singular subvariety $W^{n-1}$ of $V^{n}$ has a representative in $V_{0}^{n}$, then $\omega$ is finite at every simple point of $V^{*}$ if and only if $A=\phi\left(x_{1}, \ldots, x_{n+1}\right)$, where $\phi\left(X_{1}, \ldots, X_{n+1}\right)$ is a subadjoint polynomial of degree $\leqq m-(n+2)$.

Proof. Let $\omega=\frac{\phi\left(x_{1}, \ldots, x_{n+1}\right)}{F_{n+1}^{\prime}(x)} d x_{1} \ldots d x_{n}$, where $\phi\left(X_{1}, \ldots, X_{n+1}\right)$ is a subadjoint polynomial of degree $\leqq m-(n+2)$. Then $\omega$ is finite at every $W^{* n-1}$ which has a representative in $V_{0}^{*}$. Let $W^{* n-1}$ be any subvariety of $V^{*}$, which has no representative in $V_{0}^{*}$. Then $W^{n-1}$, the variety in $V$ which corresponds to $W^{* n-1}$, is not a singular subvariety, and $W^{*}$ has a representative in some $V_{i}^{*}(i \neq 0)$, say $i=1$.

$$
\omega=\frac{\phi\left(x_{1}, \ldots, x_{n+1}\right) \bar{x}_{1}^{m-(n+2)}}{\bar{F}_{\overline{n+1}}^{\prime}(\bar{x})} d \bar{x}_{1} \ldots d \bar{x}_{n}
$$

Since the degree of $\phi$ is $\leqq m-(n+2), \phi\left(x_{1}, \ldots, x_{n+1}\right) \bar{x}_{1}^{m-(n+2)} \in k\left[\bar{x}_{1}, \ldots\right.$, $\left.\bar{x}_{n+1}\right]$. Therefore $v_{W^{*}}\left(\phi(x) \bar{x}_{1}^{m-(n+2)}\right) \geqq 0$. Moreover since $W$ is nonsingular, $v_{W^{*}}(C)=0$. Therefore $v_{W^{*}}(\omega)=v_{W^{*}}\left(\phi(x) \bar{x}_{1}^{m-(n+2)}\right)-v_{W^{*}}(C) \gtrless 0$, which proves the corollary.

\section{$\S 3$.}

Lemma 8. Let $V^{n}$ be an affine variety in $S^{n+1}$ defined over $k$ by $F\left(X_{1}, \ldots\right.$ $\left.X_{n+1}\right)=0$ with a generic point $M=\left(x_{1}, \ldots, x_{n+1}\right)$ over $k$. We assume that $x_{n+1}$ is separably algebraic over $k\left(x_{1}, \ldots, x_{n}\right)$. Let $\omega=\frac{1}{F_{n+1}^{\prime}}\left[\sum_{i_{1}<\ldots<i_{r}} A_{i_{1} \ldots i_{r}} d x_{i_{1}} d x_{i_{2}}\right.$ $\left.\ldots d x_{i_{r}}\right]$ be a differential form on $V$ of degree $r$ defined over $k$. Then if $\omega$ is finite at every simple point of the derived normal variety $V^{*}$ of $V, A_{i_{1} \ldots i_{r}}$ are subadjoint polynomials for $V$.

Proof. This follows immediately from Theorem 3 when the degree of $\omega$ is $n$. We assume $r<n . \quad \omega=\frac{1}{F_{n+1}^{\prime}}\left[A_{1 \ldots r} d x_{1} \ldots d x_{r}+\ldots\right]$. Since $d x_{r+1}, \ldots$, $d x_{n}$ are finite at every simple point of $V^{* 10)}, \omega \cdot d x_{r+1} \ldots d x_{n}=\frac{1}{F_{n+1}^{\prime}} A_{1 \ldots r} d x_{1}$ $\ldots d x_{r} d x_{r+1} \ldots d x_{n}$ is finite at every simple point of $V^{*}$. Therefore by Theorem $3, A_{1 \ldots r}$ is subadjoint polynomial for $V$. Similarly $A_{i_{1} \ldots i_{r}}$ are subadjoint polynomials for $V$.

10) See S. Koizumi [6]. 
Notations and assumptions being same as in Theorem 4, let o) $=\frac{1}{F_{n+1}^{\prime}} \sum A_{i_{1} . . i_{r}} d x_{i_{1}} \ldots d x_{i_{r}}$, be a differential form of degree $r(<n)$ on $V$ defined-over $k$, and let $\omega$ be finite at every simple point of the derived normal variety $V^{*}$ of $V$.

$$
\begin{aligned}
& \omega=\frac{1}{F_{n+1}^{\prime}} \sum_{i_{1}<\ldots<i_{r}} A_{i_{1} \ldots i_{r}} d x_{i_{1}} \ldots d x_{i_{r}} \\
& =\frac{1}{F_{n+1}^{\prime}} \sum_{1<i_{2}<\ldots<i r} A_{1 i_{2} \ldots i_{r}} d x_{1} d x_{i_{2}} \ldots d x_{i_{r}}+\frac{1}{F_{n+1}^{\prime}} \sum_{1<j_{1}<j_{2}<\ldots<j r} A_{j_{1} \ldots j_{r}} d x_{j_{1}} \ldots d x_{j_{r}} \\
& =\frac{-1}{F_{n+1}^{\prime}} \sum_{1<i_{2}<\ldots<i_{r}} A_{1 i_{2} . . i_{r}} \bar{x}_{1}^{-2} \bar{x}_{1}^{-(r-1)} d \bar{x}_{1} d \bar{x}_{i_{2}} \ldots d \bar{x}_{i_{r}} \\
& +\frac{1}{F_{n+1}^{\prime}} \sum_{1<j_{1}<\ldots<j_{r}} A_{j_{1} \ldots j_{r}}\left(\bar{x}_{1}^{-1} d \bar{x}_{j_{1}}-\bar{x}_{j_{1}} \bar{x}_{1}^{-2} d \bar{x}_{1}\right) \ldots\left(\bar{x}_{1}^{-1} d \bar{x}_{j_{r}}-\bar{x}_{j_{r}} \bar{x}_{1}^{-2} d \bar{x}_{1}\right) \\
& =\frac{-1}{F_{n+1}^{\prime}} \sum_{1<i_{2}<\ldots<i_{r}} A_{1 i_{2} \ldots i_{r}} \bar{x}_{1}^{-(r+1)} d \bar{x}_{1} d \bar{x}_{i_{2}} \ldots d \bar{x}_{i_{r}} \\
& +\frac{1}{F_{n+1}^{\prime}} \sum_{1<j_{1}<\ldots<j r}\left[A_{j_{1} j_{2} \ldots j_{r}} \bar{x}_{1}^{-r} d \bar{x}_{j_{1}} \ldots d \bar{x}_{j_{r}}\right. \\
& \left.+\sum_{h=1}^{r} \bar{x}_{1}^{-r}\left(-\bar{x}_{j_{h}} \bar{x}_{1}^{-1}\right) d \bar{x}_{j_{1}} \ldots d \bar{x}_{1} \ldots d \bar{x}_{j_{r}}\right] \\
& =\frac{\bar{x}_{1}^{m-1}}{\bar{F}_{n+1}^{\prime}\left(\bar{x}_{1}, \ldots, \bar{x}_{n+1}\right)} \sum_{1<j_{1}<\ldots<j r} A_{j_{1} \ldots j_{r}} \bar{x}_{1}^{-r} d \bar{x}_{j_{1}} \ldots d \bar{x}_{j_{r}} \\
& -\frac{\bar{x}_{1}^{m-1}}{\bar{F}_{\overline{n+1}}^{\prime}\left(\bar{x}_{1}, \ldots, \bar{x}_{n+1}\right)}\left[\sum_{1<i_{2}<\ldots<i r} A_{1 i_{2} \ldots i_{r}} \bar{x}_{1}^{-r} \bar{x}_{1}^{-1} d \bar{x}_{1} d \bar{x}_{i_{2}} \ldots d \bar{x}_{i_{n}}\right. \\
& \left.+\sum_{1<j 1}<\ldots<j_{r} \sum_{h=1}^{r} A_{j_{1} \ldots j_{r}} \bar{x}_{1}^{-r}\left(-\bar{x}_{j_{h}} \bar{x}_{1}^{-1}\right) d \bar{x}_{j_{1}} \ldots d \bar{x}_{1} \ldots d \bar{x}_{j_{r}}\right]
\end{aligned}
$$

Therefore, by the preceding lemma $A_{j_{1} \ldots j_{r}} \bar{x}_{1}^{(m-1)-r}\left(1<j_{1}<\ldots<j_{r}\right)$ is equal to a subadjoint polynomial for $V_{1}$. Moreover

$$
A_{1 i_{2} \ldots i_{r}} \bar{x}_{1}^{(m-1)-r} \bar{x}_{1}^{-1}+\sum \pm A_{j_{1} \ldots j_{r}} \bar{x}_{1}^{m-1-r}\left(-\bar{x}_{j_{h}} \bar{x}_{1}^{-1}\right)
$$

is equal to a subadjoint polynomial for $V_{1}$. Therefore $A_{1 i_{2} \ldots i_{r}} \bar{x}_{1}^{m-1-r}+$ $\sum \pm A_{j_{1} \ldots j_{r}} \bar{x}_{1}^{m-1-r}(-1) \bar{x}_{j_{h}}$ and $A_{j_{1} \ldots j_{r}} \bar{x}_{1}^{m-1-r} \bar{x}_{j_{k}}$ are equal to a subadjoint polynomials; hence $A_{1 i_{2} \ldots i_{r}} \bar{x}_{1}^{m-1-r}$ is equal to a subadjoint polynomial. Therefore for every $i_{1}<\ldots<i_{r}, A_{j_{1} \ldots i_{r}} \bar{x}_{1}^{m-1-r}$ is equal to a subadjoint polynomial. Similarly $A_{i_{1} i_{2} \ldots i_{r}} x_{h}^{-m+1+r}$ is equal to a subadjoint polynomial for $V_{h}(1 \leqq h \leqq n)$.

As for $V_{n+1}$, by the similar argument as in the proof of Theorem 4, we can see that $A_{i_{1} \ldots i_{r}} x_{n+1}^{-(m-1-r)}$ is equal to a subadjoint polynomial for $V_{n+1}$. Therefore it follows that $A_{i_{1} \ldots i_{r}}=A_{i_{1} \ldots i_{r}}\left(x_{1}, \ldots, x_{n+1}\right)$, where $A_{i_{1} \ldots i_{r}}\left(X_{1}, \ldots\right.$, 
$\left.X_{n+1}\right)$ is of degree $\leqq m-1-r$, and $A_{i_{1} \ldots i_{r}}\left(X_{1}, \ldots, X_{n+1}\right)=0$ is a subadjoint hypersurface for $V^{n}$.

From the above argument, we see that

$$
A_{1 i_{2} \ldots i_{r}} \bar{x}_{1}^{m-1-r} \bar{x}_{1}^{-1}+\sum_{h \neq 1, i_{2}, \ldots, i_{r}} A_{h i_{2} \ldots i_{r}} \bar{x}_{h} \bar{x}_{1}^{m-1-r} \bar{x}_{1}^{-1}=\bar{A}_{i_{2} \ldots i_{r}}^{*}\left(\bar{x}_{1}, \ldots, \bar{x}_{n+1}\right)
$$

where $A_{i_{2} \ldots i r}^{*}\left(\bar{X}_{1}, \ldots, \bar{X}_{n+1}\right)=0$ are subadjoint hypersurfaces for $V_{1}$, therefore

$$
\sum_{h \neq i_{1}, \ldots, i_{r-1}} x_{h} A_{h i_{1} \ldots i_{r-1}}=A_{i_{1} \ldots i_{r-1}}^{*}\left(x_{1}, \ldots, x_{n+1}\right)^{11)},
$$

where $A_{i_{1} \ldots i_{r}}\left(X_{1}, \ldots, X_{n+1}\right)$ are subadjoint hypersurfaces for $V, A_{i_{1} \ldots i_{r}}$ are assumed skew symmetric on $i_{1}, \ldots i_{r}$.

If $x_{1}$ is separably algebraic over $k\left(x_{2}, \ldots, x_{n+1}\right)$, then $d x_{1}=-\frac{F_{2}^{\prime}}{F_{1}^{\prime}} d x_{2}$ $-\ldots-\frac{F_{n+1}^{\prime}}{F_{1}^{\prime}} d x_{n+1}$.

$$
\begin{aligned}
& (\omega)=\sum_{i_{1}<\ldots<i_{r}} \frac{A_{i_{1}} \ldots i_{r}}{F_{n+1}^{\prime}} d x_{i_{1}} \ldots d x_{i_{r}}=\sum_{1<i_{2}<\ldots<i_{r} \leqq n} \frac{A_{1 i_{3} \ldots i_{r}}}{F_{n+1}^{\prime}} d x_{1} d x_{i_{2}} \ldots d x_{i_{r}} \\
& +\sum_{1<j_{1}<\ldots<j_{r} \cong n} \frac{A_{j_{1} \ldots j_{r}}}{F_{n+1}^{\prime}} d x_{j_{1}} \ldots d x_{j_{r}} \\
& =\frac{1}{F_{1}^{\prime}}\left[\sum_{1<i_{2}<\ldots<i_{r} \leqq n}-A_{1 i_{2} \ldots i_{r}} d x_{n+1} d x_{i_{2}} \ldots d x_{i_{r}}\right. \\
& +\sum_{1<j_{1}<\ldots<j_{r} \leqq n}\left\{\sum-\varepsilon\left(\begin{array}{c}
h i_{2} \ldots i_{r} \\
j_{1} j_{2} \ldots j_{r}
\end{array}\right) A_{1 i_{2} \ldots i_{r}} \frac{F_{h}^{\prime}}{F_{n+1}^{\prime}}\right. \\
& \left.\left.+A_{j_{1} \ldots j_{r}} \frac{F_{1}^{\prime}}{F_{n+1}^{\prime}}\right\} d x_{j_{1}} \ldots d x_{j_{r}}\right] \text {. }
\end{aligned}
$$

Therefore, for $1<j_{1}<\ldots<j_{r} \leqq n$,

$$
A_{j_{1} \ldots j_{r}} \frac{F_{1}^{\prime}}{F_{n+1}^{\prime}}+\sum_{h=1}^{r}(-1)^{h} A_{1 j_{1} \ldots \hat{j}_{h} \ldots j_{r}} \frac{F_{j_{h}}^{\prime}}{F_{n+1}^{\prime}}=A_{1 j_{1} \ldots j_{r}}^{* *}\left(x_{1}, \ldots, x_{n+1}\right),
$$

where $A_{1 j_{1} \ldots j_{r}}^{* *}\left(X_{1}, \ldots, X_{n+1}\right)=0$ are subadjoint hypersurfaces of degree $m-1-r$ for $V$. This holds even if $x_{1}$ is not separably algebraic over $k\left(x_{2}, \ldots, x_{n+1}\right)$. Considering other representatives of $V$ we get

$$
\sum_{h=0}^{r}(-1)^{h} A_{i_{0} \ldots \hat{i}_{h} \ldots i_{r}-\frac{F_{i_{h}}}{F_{n+1}^{\prime}}}^{\prime}=A_{i_{0} \ldots j_{r}}^{* *}\left(x_{1}, \ldots, x_{n+1}\right)^{11)}
$$

where $A_{i_{0} \ldots i_{r}}^{* *}\left(X_{1}, \ldots, X_{n+1}\right)=0$ are subadjoint hypersurfaces for $V$.

11) This formulation is due to Y. Nakai, see [8]. 
THEOREM 5. Let $V^{n}$ be a projective variety in $L^{n+1}$. Suppose that $V^{n}$ has a representative $V_{0}$ defined over $k$ by $F\left(X_{1}, \ldots, X_{n+1}\right)=0$ with a generic point $\left(1, x_{1}, \ldots, x_{n+1}\right)$ over $k$, and $x_{n+1}$ is separably algebraic over $k\left(x_{1}, \ldots, x_{n}\right)$. Let $\omega=\frac{1}{F_{n+1}^{\prime}}\left[\sum A_{i_{1} \ldots i_{r}} d x_{i_{1}} \ldots d x_{i_{r}}\right]$ be a differential form of degree $r(<n)$ on $V$ defined over $k$, which is finite at every simple point of the derived normal variety $V^{*}$ of $V$. Then $A_{i_{1} \ldots i_{r}}=A_{i_{1} \ldots i_{r}}\left(x_{1}, \ldots, x_{n+1}\right)$ where $A_{i_{1} \ldots i_{r}}\left(X_{\llcorner}, \ldots, X_{n+1}\right)=0$ are subadjoint hypersurfaces of degree $m-1-r$ for $V, m$ being the degree of $F\left(X_{1}, \ldots, X_{n+1}\right)$. Furthermore $(*)$ and $(* *)$ also hold.

Lemma 9. Let $V^{n}$ in $\mathrm{S}^{n+1}$ be a generic projection of a normal variety $\bar{V}^{n}$. Let $F\left(X_{1}, \ldots, X_{n+1}\right)=0$ be equation for $V$ and let $\omega=\frac{1}{F_{n+1}^{\prime}} \sum A_{i_{1} \ldots i_{r}} d x_{i_{1}} \ldots d x_{i_{r}}$ be a differential form on $V^{n}$, such that $A_{i_{1} \ldots i_{r}}$ are subadjoint polynomials for $V$ for every $\left(i_{1}, \ldots, i_{r}\right)$ and $(* *)$ holds. Then $\omega$ is finite at every simple point of $\bar{V}$.

Proof. Let $k$ be a field of definition of $\bar{V}^{n}$ in $S^{m}$. Since $V^{n}$ is a generic projection of $\bar{V}^{n}$,

$$
x_{i}=u_{0 i}+\sum_{j=1}^{m} u_{j i} y_{j} \quad(i=1, \ldots, n+1)
$$

where $u_{j i}, j=0, \ldots, m ; i=1, \ldots, n+1$ are $(n+1)(m+1)$ independent variables over $k$ and $\left(y_{1}, \ldots, y_{m}\right)$ is a generic point of $\bar{V}^{n}$ over $k(u)=k\left(u_{j i}\right)$. $\left(x_{1}, \ldots, x_{n+1}\right)$ is a generic point of $V^{n}$ over $k(u)$.

Since $\left(y_{1}, y_{2}, \ldots, y_{m}\right)$ is of dimension $n$ over $k(u)$, we see that $\left(x_{1}, \ldots\right.$, $\left.x_{n-1}\right)$ are independent over $k(u)$. We consider the linear variety $H^{m-(n-1)}$ defined by

$$
\begin{gathered}
-x_{1}+u_{01}+u_{11} Y_{1}+\ldots+u_{m 1} Y_{m}=0 \\
\ldots \ldots \\
-x_{n-1}+u_{0 n-1}+u_{1 n-1} Y_{1}+\ldots+u_{m n-1} Y_{m}=0
\end{gathered}
$$

Then since $\bar{V}^{n}$ is normal defined over $k$ and $H$ is independent over $k, H \cdot \bar{V}^{n}$ is irreducible and is also normal. ${ }^{12)}$ Further $H \cdot \bar{V}^{n}$ is defined over $k\left(\left(u^{\prime}\right), x_{1}\right.$, $\left.\ldots, x_{n-1}\right)$, of dimension 1 and $\left(y_{1}, \ldots, y_{m}\right)$ is a generic point of $H \cdot \bar{V}^{n}$ over $k\left(u, x_{1}, \ldots, x_{n-1}\right)$, where $\left(u^{\prime}\right)=\left(u_{01}, \ldots, u_{m 1}, \ldots, u_{0 n-1}, \ldots, u_{m n-1}\right)$.

Let $W^{n-1}$ be a non-singular subvariety of $\bar{V}$ which is algebraic over $k(u)$. By $W$ we can introduce the valuation $v_{w}$ of $\overline{k(u)}\left(y_{1}, \ldots, y_{m}\right)$, of dimension

12) A. Seidenberg [9]. 
$n-1$ over $\overline{k(u)}$. We may assume without loss of generality that $v_{W}$ is the valuation over $\overline{k(u)}\left(x_{1}, \ldots, x_{n-1}\right)$, of dimension 0 . For, $v_{w}$ is the valuation over the field $K$ which is adjointed certain $n-1$ elements, say $x_{3}, \ldots, x_{n+1}$, among $x_{1}, \ldots, x_{n+1}$, over $\overline{k(u)}$. Then by $(* *)$ we get

$$
\omega=\frac{1}{F_{1}^{\prime}} \sum B_{j_{1} \ldots j r} d x_{j_{1}} \ldots d x_{j r}
$$

where $B_{j_{1} \ldots j r}$ are subadjoint polynomials, and therefore we can make the same argument as in the case $K=\overline{k(u)}\left(x_{1}, \ldots, x_{n+1}\right)$. Let $\bar{v}$ be an extension of $v_{W}$ to the field $k(u)\left(x_{1}, \ldots, x_{n-1}\right)\left(y_{1}, \ldots, y_{m}\right)$ and let $t$ be an element in $k(u)\left(x_{1}, \ldots, x_{n-1}\right)\left(y_{1}, \ldots, y_{m}\right)$ such that $\ddot{v}(t)=1$ and let $y_{i}=\alpha_{i}+\beta_{i} t+\ldots$, $\alpha_{i}, \beta_{i}$ belong to $\overline{k(u)}\left(x_{1}, \ldots, x_{n-1}\right)$. Since any points of $\bar{V} \cdot H$ are simple and $\left(\alpha_{1}, \ldots, \alpha_{m}\right)$ is a point of $\bar{V} \cdot H$, we see that $\beta_{i} \neq 0$ for at least one $i$ and we can assume that $t \in k\left(\alpha_{1}, \ldots, \alpha_{m}\right)\left(y_{1}, \ldots, y_{m}\right)$. Now the restriction of $\bar{v}$ to $k\left(\left(u^{\prime}\right), x_{1}, \ldots, x_{n-1}\right)\left(\alpha_{1}, \ldots, \alpha_{m}\right)\left(y_{1}, \ldots, y_{m}\right)$ is a valuation of dimension 0 over $\overline{\left.k\left(\overline{\left(u^{\prime}\right.}\right), x_{1}, \ldots, x_{n-1}\right)\left(\alpha_{1}, \ldots, \alpha_{m}\right)}$. Therefore all $\beta_{i}$ belong to $k\left(\left(u^{\prime}\right), x_{1}, \ldots, x_{n-1}\right)\left(\alpha_{1}, \ldots, \alpha_{m}\right)$. As $\bar{V} \cdot H$ is defined over $k\left(\left(u^{\prime}\right), x_{1}, \ldots\right.$, $\left.x_{n-1}\right)$ of dimension 1 , the dimension of $\left(\alpha_{1}, \ldots, \alpha_{m}\right)$ over $k\left(\left(u^{\prime}\right), x_{1}, \ldots\right.$, $\left.x_{n-1}\right)$ is at most 1 , hence the dimension of $\left(\beta_{1}, \ldots, \beta_{m}\right)$ over $k\left(\left(u^{\prime}\right), x_{1}, \ldots\right.$, $\left.x_{n-1}\right)$ is also at most 1 . Moreover since $\left(x_{1}, \ldots, x_{n-1}\right)$ are independent over $k(u),\left(u_{0 n}, \ldots, u_{m n}, u_{0 n+1}, \ldots, u_{m n+1}\right)$ are independent over $k\left(\left(u^{\prime}\right), x_{1}, \ldots\right.$, $\left.x_{n-1}\right)$, therefore for at least one $i(i=n$ or $n+1)$, say $i=n,\left(u_{0 n}, \ldots, u_{m n}\right)$ is a set of independent elements over $k\left(\left(u^{\prime}\right), x_{1}, \ldots, x_{n-1}\right)\left(\alpha_{1}, \ldots, \alpha_{m}\right)$. Since

$$
\begin{aligned}
x_{n} & =u_{0 n}+u_{1 n} y_{1}+\ldots+u_{m n} y_{m} \\
& =\left(\sum_{j=0}^{m} u_{j n} \alpha_{j}\right)+\left(\sum_{j=0}^{m} u_{j n} \beta_{j}\right) t+\ldots
\end{aligned}
$$

and since $\beta_{j}$ belong to $\overline{k\left(\left(u^{\prime}\right), x_{1}, \ldots, x_{n-1}\right)\left(\alpha_{1}, \ldots, \alpha_{m}\right)}$, it follows that $\sum_{j=0}^{m} u_{j n} \beta_{j} \neq 0$, which shows that there is an element $A$ in $\overline{k(u)\left(x_{1}, \ldots, x_{n-1}\right)}$ such that $\bar{v}\left(x_{n}-A\right)=1$. Therefore the different of $\overline{k(u)} \overline{\left(x_{1}, \ldots, x_{n-1}\right)}\left(y_{1}, \ldots\right.$, $\left.y_{m}\right)$ with respect to $\overline{k(u)\left(x_{1}, \ldots, x_{n-1}\right)}\left(x_{n}\right)$ is not divisible by $\bar{v}$. Since $k(u)\left(x_{1}, \ldots, x_{n-1}\right)\left[y_{1}, \ldots, y_{m}\right]$ and $k(u)\left(x_{1}, \ldots, x_{n-1}\right)\left[y_{1}, \ldots, y_{m}\right]$ are integrally closed, we see that the different of $k(u)\left(x_{1}, \ldots, x_{n-1}\right)\left(y_{1}, \ldots, y_{m}\right)$ $=k(u)\left(x_{1}, \ldots, x_{n+1}\right)$ with respect to $k(u)\left(x_{1}, \ldots, x_{n-1}\right)\left(x_{n}\right)$ is not divisible by $v_{w}$. Therefore, by the same argument as in the proof of Lemma 7, since 
$F_{n+1} \cdot \mathfrak{D}=\mathrm{c}$, we get $v_{w}\left(F_{n+1}\right)=v_{w}(C)$, where $C$ is the subadjoint divisor for $V$. Since $A_{i_{1} \ldots i_{r}}$ are subadjoint polynomials, $\omega$ is finite at $W$. If $W$ is not algebraic over $k(u)$, then obviously $\omega$ is finite at $W$. Therefore $\omega$ is finite at every nonsingular $W$ of $\bar{V}$, and hence $\omega$ is finite at every simple point of $\bar{V}$.

Theorem 6. ${ }^{13)}$ Let $V^{n}$, in projective $n+1$ space, be a generic projection of a normal projective variety $\dot{V}^{n}$. Let $\left(1, x_{1}, \ldots, x_{n+1}\right)$ be a generic point of $V_{0}$ and let $F\left(X_{1}, \ldots, X_{n+1}\right)=0$ be equation for $V_{0}$. Let $\omega=\frac{1}{F_{n+1}^{\prime}} \sum A_{i_{1} \ldots i_{r}} d x_{i_{1}}$ $\ldots d x_{i_{r}}$ be a differential form on $V$. Then, if $A_{i_{1} \ldots i_{r}}=A_{i_{1} \ldots i_{r}}\left(x_{1}, \ldots, x_{n+1}\right)$ are subadjoint polynomials of degree $m-1-r$ and moreover $(*)$ and $(* *)$ hold, $\omega$ is finite at every simple point of $\bar{V}$ and conversely.

Proof. Let $W^{*}$ be a subvariety of $\bar{V}$ and let $W$ be the corresponding subvariety of $V$. Then, if $W$ has a representative in $V_{0}, \omega$ is finite at $W^{*}$ by the preceding lemma. As for $V_{1}$ since $(*)$ hold, we see by the proof of Theorem 5 , that

$$
\omega=\frac{1}{\bar{F}_{\bar{n}+1}^{\prime}} \sum B_{i_{1} \ldots i_{r}} d \bar{x}_{i_{1}} \ldots d \bar{x}_{i_{r}}
$$

where $B_{i_{1} \ldots i_{r}}$ are equal to polynomials on $\bar{x}_{1}, \ldots, \bar{x}_{n+1}$. Therefore $(\omega)$ is finite at every non-singular $W^{n-1}$ of $V_{1}$, similarly at every non-singular $W^{n-1}$ of $V_{i}$. As $V$ is a generic projection of $\bar{V}$, all singular $W^{n-1}$ of $V$ have representatives in $V_{0}$, hence $\omega$ is finite at every simple point of $\bar{V}$. The converse follows immediately from Theorem 5 , since $\bar{V}$ is biregularly birationally equivalent to a variety derived from $V$ by normalization.

COROLlary. In the above theorem, if $\bar{V}$ is a variety without singularity, then $\omega$ is of the first kind.

Corollary. Assumptions being as in the above theorem, let $\omega$ $=\sum_{n=0}^{n}(-1)^{h+1} \frac{A_{h}}{F_{n+1}^{\prime}} d x_{1} \ldots \widehat{d x}_{h} \ldots d x_{n}$ be a differential form on $V^{n}$, where $A_{1}, \ldots, A_{n}$ and $A_{n+1}=\frac{A_{1} F_{1}^{\prime}+\ldots+A_{n} F_{n}^{\prime}}{F_{n+1}^{\prime}}$ are equal to subadjoint poly. nomials of degree $m-n$ and moreover

$$
A_{1}=x_{1} \theta=\psi_{1}, A_{2}=x_{2} \theta+\psi_{2}, \ldots, A_{n+1}=x_{n+1} \theta+\psi_{n+1},
$$

13) SeeY. Nakai [8]. 
where $\phi_{1}, \psi_{2}, \ldots, \psi_{n+1}$ polynomials of degree $<m-n$. Then $\omega$ is finite at every simple point of $\bar{V}$, and conversely.

$\S 4$. In this section we always assume that the characteristic of the field $k$ is 0 and give a proof of the following

THEOREм 7. Let $V^{r}(r \gg 2)$ be a projective normal variety defined over a field $k$ of characteristic 0 and let $\omega_{1}, \ldots, \omega_{s}$ be linearly independent simple closed differential forms which are finite at every simple point of $V$. Then the induced forms $\omega_{1}^{\prime}, \ldots, \omega_{s}^{\prime}$ on a generic hyperplane section (over $k$ ) $W^{r-1}$ are also linearly independent.

Let $V^{r}$ be in the projective space $L^{n}$ and let $W^{r-1}$ be the intersection of $V^{r}$ and the hyperplane $u_{0} X_{0}+u_{1} X_{1}+\ldots+u_{n} X_{n}=0$, where $u_{0}, \ldots, u_{n}$ are $n+1$ independent elements over $k$. It is well known that $W^{r-1}$ is also a normal variety defined over $k\left(u_{0}, u_{1}, \ldots, u_{n}\right)$. First we show that it is enough that we treat the case where all the $\omega_{1}, \ldots, \omega_{s}$ are defined over $k$. If $\omega_{1}, \ldots, \omega_{s}$ are defined over a certain larger field $K$, we can express $\omega_{i}$ in the following form

$$
\omega_{i}=\sum_{j} \alpha_{j} \omega_{i j} \quad i=1, \ldots, s,
$$

where $\alpha_{j}$ are constants defined over $K$ and $\omega_{i j}$ are simple closed differential forms which are finite at every simple point of $V$, defined over $k^{14}$ ) Choosing among the $\omega_{i j}$ a maximal set of linearly independent ones $\bar{\omega}_{h}(h=1, \ldots, t)$, and expressing all $\omega_{i j}$ in terms of these, we get for $\omega_{i}$ an expression of the form

$$
\omega_{i}=\sum_{h=1}^{t} \beta_{i h} \bar{\omega}_{h}(i=1, \ldots, s) \text {, where } \beta_{i h} \text { are constants. }
$$

As $\omega_{i}(i=1, \ldots, s)$ and $\bar{\omega}_{h}(h=1, \ldots, t)$ are linearly independent respectively, we see that the rank of the matrix $\left(\beta_{i h}\right)$ is $s$. Let $\bar{\omega}_{h}^{\prime}$ be the induced forms of $\bar{\omega}_{h}$ on $W$. Then $\omega_{i}^{\prime}=\sum_{h} \beta_{i h} \bar{\omega}_{h}^{\prime}$; therefore if $\bar{\omega}_{h}^{\prime}$ are linearly independent, $\omega_{i}^{\prime}$ are also linearly independent, which shows that we can assume that $\omega_{i}$ are all defined over $k$.

Next we show that we may assume $r=2$. Let $r>2$ and let $\omega_{1}^{\prime}, \ldots, \omega_{s}^{\prime}$ be not linearly independent. As $\omega_{i}$ is defined over $k, \omega_{i}^{\prime}$ is defined over $k\left(u_{0}\right.$,

14) See S. Koizumi [6]. 
$\left.u_{1}, \ldots, u_{n}\right)$, therefore there must be a linear equation

$$
\sum \alpha_{i} \omega_{i}^{\prime}=0
$$

with $\alpha_{i}$ (not all zero) constants defined over $k\left(u_{0}, \ldots, u_{n}\right)$; namely there is a differential form $\Omega=\sum \alpha_{i} \omega_{i}$ on $V$ which is finite at every simple point of $V$ and induces 0 on $W$. Now let $\left(v_{0}, \ldots, v_{n}\right)$ be a set of independent variables over $k\left(u_{0}, \ldots, u_{n}\right)$ and consider the intersection $V_{(\mathbf{i})}$ of $V$ and the hyperplane $v_{0} X_{0}+v_{1} X_{1}+\ldots+v_{n} X_{n}=0$ and the intersection $W_{(1)}$ of $W$ and the (generic) hyperplane $v_{0} X_{0}+v_{1} X_{1}+\ldots+v_{n} X_{n}=0$.

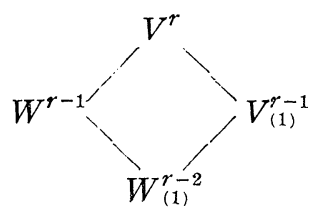

Then $W_{(1)}$ is a simple subvariety of $V$ and as $\Omega$ induces 0 on $W, \Omega$ induces on $W_{(1)}$ also 0 . Since $\Omega$ is defined over $k\left(u_{0}, \ldots, u_{n}\right)$ and $V_{(1)}$ is a generic hyperplane section over $k\left(u_{0}, \ldots, u_{n}\right)$ of $V$, the form $\Omega_{(1)}$ induced by $\Omega$ on $V_{(1)}$ is not 0 , and is finite at every simple point of $V_{(1)}$ and closed. But as $\Omega$ induces on $W_{(1)} 0, \Omega_{(1)}$ must induces 0 on $W_{(1)}$ which is a generic hyperplane section of $V_{(1)}$. Therefore by induction we can assume $r=2$.

Next we consider the generic projection. We choose $4(n+1)$ elements $v_{i}^{(0)}$, $v_{i}^{(1)}, v_{i}^{(2)}, v_{i}^{(3)},(i=0, \ldots, n)$ independent over $k$ and 4 elements $w_{0}, w_{1}, w_{2}, w_{3}$ independent over $k\left(\left(v_{i}^{(0)}\right), \ldots,\left(v_{(i)}^{(3)}\right)\right)$ which satisfy the equations

$$
\begin{gathered}
w_{0} v_{i}^{(0)}+w_{1} v_{i}^{(1)}+w_{2} v_{i}^{(2)}+w_{3} v_{i}^{(3)}=u_{i} \\
i=0,1, \ldots, n
\end{gathered}
$$

Let $\left(y_{0}, y_{1}, \ldots, y_{n}\right)$ be homogeneous coordinates of a generic point of $V^{2}$ over $k\left(\left(v_{i}^{(0)}\right), \ldots,\left(w_{i}\right)\right)$ and put $\bar{y}_{0}=v_{0}^{(0)} y_{0}+v_{1}^{(0)} y_{1}+\ldots+v_{n}^{(0)} y_{n}, \ldots, \bar{y}_{3}=v_{0}^{(3)} y_{0}$ $+\ldots+v_{n}^{(3)} y_{n}$ and consider the projective variety $\bar{V}^{2}$ in $L^{3}$ with the generic point over $k\left(\left(v_{i}^{(0)}\right), \ldots,\left(w_{i}\right)\right)$, whose homogeneous coordinates are $\left(\bar{y}_{0}, \bar{y}_{1}, \bar{y}_{2}\right.$, $\left.\bar{y}_{3}\right)$. This variety is birationally equivalent to $V^{2}$ and by this birational correspondence the generic hyperplane section $W^{1}$, the intersection of $V$ and the hyperplane $u_{0} X_{0}+\ldots+u_{n} X_{n}=0$, correspond to the generic hyperplane section $\bar{W}^{1}$, the intersection of $\bar{V}^{2}$ and the hyperplane $w_{0} \bar{X}_{0}+w_{1} \bar{X}_{1}+w_{2} \bar{X}_{2}+w_{3} \bar{X}_{3}=0$, and furthermore $W^{1}$ and $\bar{W}^{1}$ are birationally equivalent. Therefore if $\Omega$ induces 
0 on $W, \Omega$ also induces 0 on $\bar{W}$. Considering the non-homogeneous coordinates

$$
(1, x, y, z)=\left(\bar{y}_{0} / \bar{y}_{0}, \bar{y}_{1} / y_{0}, \bar{y}_{2} / \bar{y}_{0}, \bar{y}_{3} / \bar{y}_{0}\right)
$$

we get

$$
w_{0}+w_{1} x+w_{2} y+w_{3} z=0 .
$$

Now, since $\bar{V}^{2}$ is of dimension 2 in $L^{3}$, its equation in non-homogeneous coordinates is

$$
F(x, y, z)=0
$$

whose order we assume to be $m$. Let us consider a differential form

$$
\omega=R d x+S d y
$$

which is finite at every simple point of $V$. Then we get

$$
S=\frac{A}{F_{z}^{\prime}}, \quad R=-\frac{B}{F_{z}^{\prime}}, \quad C=-\frac{A F_{x}^{\prime}+B F_{y}^{\prime}}{F_{z}^{\prime}}
$$

where $A, B, C$ are subadjoint polynomials in $(x, y, z)$ of degree $\leqq m-2$ and we have a relation

$$
A F_{x}^{\prime}+B F_{y}^{\prime}+C F_{z}^{\prime}=D F
$$

where $D$ is a polynomial of degree $\leqq m-3$.

Lemma. (Castelnuovo) $)^{15)}$ Let $f(X, Y, Z)=0$ be an irreducible plane curve of degree $m$ without singular points except nodes. Let

$$
A f_{X}^{\prime}+B F_{Y}^{\prime}+C f_{Z}^{\prime}=0
$$

where $A, B, C$ are forms in $X, Y, Z$ of degree $l$. Then if $l<m-1$, they must be identically zero.

Proof. Let $k$ be a field containing the coefficients of $f, A, B$ and $C$ and let $u_{0}, u_{1}, u_{2}$ be a set of independent elements over $k$. Let $D$ be the curve defined by $u_{0} f_{X}^{\prime}+u_{1} f_{Y}^{\prime}+u_{2} f_{Z}^{\prime}=0$. Then by the classical Bertini's theorem, $D$ has no multiple points except the points which satisfy $f_{X}^{\prime}=f_{Y}^{\prime}=f_{Z}^{\prime}=0$. But since the latter points are nodes of $f=0$, they must be the simple points of $D$. Therefore $D$ has no multiple points and therefore $D$ is an irreducible curve of degree $m-1$.

15) F, Severi [10]. 
Let $P_{1}, \ldots, P_{d}$ be all nodes of $f=0$ and denote the cycle $P_{1}+\ldots+P_{d}$ $=H$. Let $\xi$ be an adjoint curve of degree $m-3$ for $f=0$ (a curve passing through $\left.P_{1}, \ldots, P_{d}\right)$ and let $K=D \cdot \xi-H$ be cycle on $L^{2}$, where $D \cdot \xi$ is the intersection of $D$ and $\xi$. $D \cdot \xi$ and $K$ are positive divisors on $D$. Since $D$ is withought singularity all curves of degree $m-3$ cut out on $D$ a complete linear series containing $D \cdot \xi$ which is non-special. The number of linearly independent curves of degree $m-3$ in $L^{2}$ is $\frac{(m-1)(m-2)}{2}$, and that of linearly independent curves of degree $m-3$ passing $P_{1}, \ldots, P_{d}$, which is the genus of $f=0$, is $\frac{(m-1)(m-2)}{2}-d$, namely the dimension of the latter linear series is equal to that of the former series minus the degree of $H$. Since the former is non-special we get, by the Riemann-Roch theorem, or Brill-Noether's Reduction theorem, that the latter series, and hence $K$ is non-special.

Now Suppose for a moment that there exists an adjoint curve $\eta$ of degree $m-1$ for $f=0$, and a curve $\varphi$ of degree $m-2$, such that $\varphi \cdot D>G=\eta \cdot D-H$. Let $\varphi \cdot D=G+S$. Then $S$ is positive and its degree is $(m-1)(m-2)$ $-(m-1)(m-1)+d=\frac{(m-1)(m-4)}{2}-$ genus of $f>\frac{(m-2)(m-3)}{2}$. Therefore there is a curve $\psi$ of degree $m-4$ passing the points of $S$, namely $\psi \cdot D$ $>S$. Let $\psi \cdot D=S+T$ and let $R$ be the intersection of a line and $D$. Then

$$
H+K \sim(m-3) R, G+H \sim(m-1) R, G+S \sim(m-2) R, S+T \sim(m-4) R .
$$

Therefore $K$ and $T$ are linearly equivalent, but this is a contradiction, because $T$ is special since there is a curve of degree $m-4$ ( = an adjoint curve of $(m-1)-3$ for $D$ ) passing the points of $T$, on the other hand $K$ is non-special.

Therefore there is no curve $\varphi$ of degree $m-2$ such that $\varphi \cdot D>G=\eta \cdot D$ $-H$, where $\eta$ is an adjoint curve of degree $m-1$ for $f=0$.

Now suppose

$$
A f_{X}^{\prime}+B f_{Y}^{\prime}+C f_{Z}^{\prime}=0
$$

where $A, B$ and $C$ are forms of degree $l<m-1$, not all zero, say $A \neq 0$. Then

$$
A\left(u_{0} f_{X}^{\prime}+u_{1} f_{Y}^{\prime}+u_{z} f_{Z_{1}}^{\prime}\right)+\left(u_{0} B-u_{1} A\right) f_{Y}^{\prime}+\left(u_{0} C-u_{2} A\right) f_{Z}^{\prime}=0 .
$$

The point of the intersection of $D$ and $f_{Y}^{\prime}=0$ which is not in $H$ i.e. the point which does not satisfy $f_{Z}^{\prime}=0$, must be contained in the curve $u_{0} C-u_{2} A$ of degree $\leqq m-2$; this is a contradiction. 
Let $\bar{F}\left(\bar{Y}_{0}, \bar{Y}_{1}, \bar{Y}_{2}, \bar{Y}_{3}\right)=0$ be the homogeneous equation of $\bar{V}$, and let $l$ be the maximum of the degrees of $A, B$ and $C$. Then the degree of $D$ is $\leqq l-1$, and we get from (1)

$$
\bar{A} \bar{F}_{\bar{Y}_{1}}^{\prime}+\bar{B} \bar{F}_{\bar{Y}_{2}}^{\prime}+\bar{C} \bar{F}_{\bar{Y}_{3}}^{\prime}=\bar{D} \bar{F}
$$

where $\bar{A}, \bar{B}$ and $\bar{C}$ are of degree $l$ and $\bar{D}$ is of degree $l-1$. Since $\bar{Y}_{0} \bar{F}_{\bar{Y}_{0}}^{\prime}$ $+\bar{Y}_{1} \bar{F}_{\bar{Y}_{1}}^{\prime}+\bar{Y}_{2} \bar{F}_{\bar{Y}_{2}}^{\prime}+\bar{Y}_{3} \bar{F}_{\bar{Y}_{3}}^{\prime}=m \bar{F}$, we get

$$
\left(m \bar{A}-\bar{Y}_{1} \bar{D}\right) \bar{F}_{\bar{Y}_{1}}^{\prime}+\left(m \bar{B}-\bar{Y}_{2} \bar{D}\right) \bar{F}_{\bar{Y}_{2}}^{\prime}+\left(m \bar{C}-\bar{Y}_{3} \bar{D}\right) \bar{F}_{\bar{Y}_{3}}^{\prime}=\bar{Y}_{0} \bar{D} \bar{F}_{\bar{Y}_{0}}^{\prime}
$$

As $\bar{V}$ is a generic projection of normal variety, the intersection of $\bar{V}$ and $\bar{Y}_{0}=0$ is an algebraic plane curve without singular points except nodes. From the above lemma we see that $m \bar{A}$ and $\bar{Y}_{1} \bar{D}$ become equal if we put $\bar{Y}_{0}=0$ etc., therefore the homogeneous term of degree $l$ of $m A$ is equal to that of $X D$, etc., hence we get

$$
A=x \theta+A_{1}, B=y \theta+B_{1}, C=z \theta+C_{1}, D=m \theta+D_{1}
$$

where $\theta$ is a homogeneous polynomial of degree $l-1$ and $A_{1}, B_{1}, C_{1}$ are of degree $\leqq l-1$ and $D_{1}$ is of degree $\leqq l-2$. Now we see that if $\omega$ is further closed, then $A, B, C$ are polynomials of degree exactly $m-2$. For, suppose its degree $l<m-2$. From (1),

$$
\begin{gathered}
d w=\frac{A_{x}^{\prime}+B_{y}^{\prime}+C_{z}^{\prime}-D}{F_{z}^{\prime}} d x d y \\
A_{x}^{\prime}=\theta+x \theta_{x}^{\prime}+A_{1 x}^{\prime}, \quad B_{y}^{\prime}=\theta+y \theta_{y}^{\prime}+B_{1 y}^{\prime}, \quad C_{z}^{\prime}=\theta+z \theta_{z}^{\prime}+C_{1 z}^{\prime} \\
A_{x}^{\prime}+B_{y}^{\prime}+C_{z}^{\prime}-D=(3-m) \theta+x \theta_{x}^{\prime}+y \theta_{y}^{\prime}+z \theta_{z}^{\prime}+\left(A_{1 x}^{\prime}+B_{1 y}^{\prime}+C_{1 z}^{\prime}-D_{1}\right) \\
=(l+2-m) \theta+\text { term of degree } \leqq l-2 \\
\neq 0 .
\end{gathered}
$$

From above we get the following

Lemma 10. Let $\omega=R d x+S d y$ be a closed differential form on $V$, which is finite at every simple point of $V$. Then we get $S=\frac{A}{F_{z}^{\prime}}, R=-\frac{B}{F_{z}^{\prime}}$ where $A$, $B$ are polynomials in $(x, y, z)$ of degree exactly $m-2$.

Now we proceed to prove the theorem. $\Omega=\sum \alpha_{i} \omega_{i}$ may be expressible in the following form 


$$
\Omega=\sum_{h \in 1} r h \bar{\omega} h
$$

(with some index set I) where $\bar{\omega}_{h}$ are the closed differential forms, finite at every simple point of $V$, defined over $k\left(\left(v_{i}^{(0)}\right), \ldots,\left(v_{i}^{(3)}\right)\right)$ on $\bar{V}^{2}$ and $\gamma_{h}$ are constant functions defined over $k\left(\left(v_{i}^{(0)}\right), \ldots,\left(v_{i}^{(3)}\right),\left(w_{i}\right)\right)$. Further we may assume without loss of generality that $\gamma_{h}$ are monomials in $w_{0}, w_{1}, w_{2}, w_{3}$ and $\bar{\omega}_{h} \neq 0$ for all $h \in \mathrm{I}$. Let $\bar{\omega}_{h}=R_{h} d x+S_{h} d y$ and let $S_{h}=\frac{A_{h}}{F_{z}^{\prime}}, \quad R_{h}=-\frac{B_{h}}{F_{z}^{\prime}}$. Then $\Omega=R d x+S d y=-\frac{B}{F_{z}^{\prime}} d x+\frac{A}{F_{z}^{\prime}} d y$, where $R=\sum \gamma_{h} R_{h}, \quad S=\sum r_{h} S_{h}$ and $A=\sum r_{h} A_{h}, B=\sum \gamma_{h} B_{h}$. Let $\left(x^{\prime}, y^{\prime}, z^{\prime}\right)$ be a (non-homogeneous) generic point of $\bar{W}^{1}\left(=\right.$ the intersection of $\bar{V}^{2}$ and the generic hyperplane $w_{0} X_{0}+w_{1} \mathrm{X}_{1}+w_{2} X_{2}$ $\left.+w_{3} X_{3}=0\right)$ over $k\left(\left(v_{i}^{(0)}\right), \ldots,\left(v_{i}^{(3)}\right),\left(w_{i}\right)\right)$. The induced differential form $\Omega^{\prime}$ of $\Omega$ on $\bar{W}^{1}$ is by definition

$$
\Omega^{\prime}=R^{\prime} d x^{\prime}+S^{\prime} d y^{\prime}=\sum \gamma_{h} R_{h}^{\prime} d x^{\prime}+\sum \gamma_{h} S_{h}^{\prime} d y^{\prime},
$$

where $R^{\prime}, S^{\prime}, R_{h}^{\prime}$ and $S_{h}^{\prime}$ are the specialization of $R, S, R_{h}$ and $S_{h}$ over the specialization $(x, y, z) \rightarrow\left(x^{\prime}, y^{\prime}, z^{\prime}\right)$ with respect to $k\left(\left(v_{i}^{(0)}\right), \ldots,\left(v_{i}^{(3)}\right),\left(w_{i}\right)\right)$ respectively. Since

$$
d z=-\frac{F_{x}^{\prime}}{F_{z}^{\prime}} d x-\frac{F_{y}^{\prime}}{F_{z}^{\prime}} d y
$$

we have

$$
d z^{\prime}=-\left(\frac{F_{x}^{\prime}}{F_{z}^{\prime}}\right)^{\prime} d x^{\prime}-\left(\frac{F_{y}^{\prime}}{F_{z}^{\prime}}\right)^{\prime} d y^{\prime}
$$

where $\left(\frac{F_{x}^{\prime}}{F_{z}^{\prime}}\right)^{\prime}$ and $\left(\frac{F_{y}^{\prime}}{F_{z}^{\prime}}\right)^{\prime}$ are the specializations of $\frac{F_{x}}{F_{z}^{\prime}}$ and $\frac{F_{y}^{\prime}}{F_{z}^{\prime}}$ respectively over $(x, y, z) \rightarrow\left(x^{\prime}, y^{\prime}, z^{\prime}\right)$ with respect to $k\left(\left(v_{l}^{(0)}\right), \ldots,\left(v_{i}^{(3)}\right),\left(w_{i}\right)\right)$. Moreover as $w_{0}+w_{1} x^{\prime}+w_{2} y^{\prime}+w_{3} z^{\prime}=0$

$$
w_{1} d x^{\prime}+w_{2} d y^{\prime}+w_{3} d z^{\prime}=0 .
$$

Therefore

$$
d x^{\prime}=-\frac{w_{2}-w_{3}\left(\frac{F_{y}^{\prime}}{F_{z}^{\prime}}\right)^{\prime}}{w_{1}-w_{3}\left(\frac{F_{x}^{\prime}}{F_{z}^{\prime}}\right)^{\prime}} d y^{\prime}
$$




$$
=\left(-R^{\prime} \frac{w_{2}-w_{3}\left(\frac{F_{y}^{\prime}}{F_{z}^{\prime}}\right)^{\prime}}{w_{1}-w_{3}\left(\frac{F_{x}^{\prime}}{F_{z}^{\prime}}\right)^{\prime}}+S^{\prime}\right) d y^{\prime} .
$$

If we suppose $\Omega^{\prime}=0$, as $y^{\prime}$ is a variable on $\bar{W}^{1}$ over $k\left(\left(v_{i}^{(0)}\right), \ldots,\left(v_{i}^{(3)}\right),\left(w_{i}\right)\right)$, we get

$$
-R^{\prime} \frac{w_{2}-w_{3}\left(\frac{F_{y}^{\prime}}{F_{z}^{\prime}}\right)^{\prime}}{w_{1}-w_{3}\left(\frac{F_{x}^{\prime}}{F_{z}^{\prime}}\right)^{\prime}}+S^{\prime}=0 .
$$

Therefore if we put $D=S\left(w_{1}-w_{3} \frac{F_{x}^{\prime}}{F_{z}^{\prime}}\right)-R\left(w_{2}-w_{3} \frac{F_{y}^{\prime}}{F_{z}^{\prime}}\right)$, since the function $\frac{D}{w_{1}-w_{3} \frac{F_{x}^{\prime}}{F_{z}^{\prime}}}$ is zero on $\bar{W}^{1}$, the function $D$ must be zero on $\bar{W}^{1}$; and $E=w_{1} A$ $+w_{2} B+w_{3} C$ must be zero on $\bar{W}$, where $A=\sum \gamma_{h} A_{h}, B=\sum \gamma_{h} B_{h}, C=\sum \gamma_{h} C_{h}$ $C_{h}=-\frac{-A_{h} F_{x}^{\prime}+B_{h} F_{y}^{\prime}}{F_{z}^{\prime}}$.

As $\bar{W}^{1}$ is a generic hyperplane section of $\bar{V}$, the corresponding prime ideal to $\bar{W}$ is generated by $F$ and $\left(w_{0}+w_{1} X+w_{2} Y+w_{3} Z\right){ }^{16)}$ where we can assume without loss of generality that $F$ is of order $m$ on $Z$. Since $E$ is 0 on $\bar{W}, E$ belongs to this ideal, namely if we replace $w_{0}$ to $-\left(w_{1} X+w_{2} Y+w_{3} Z\right)$ in $E$, then $E$ becomes a polynomial $\bar{E}$ which is divisible by $F$. From $\gamma_{h}$ we choose

$$
w_{0}^{\lambda} w_{2}^{\mu} w_{3}^{\nu}, w_{0}^{\lambda-1} w_{1} w_{2}^{u} w_{3}^{\nu}, \ldots, w_{0} w_{1}^{\lambda-1} w_{2}^{\mu} w_{3}^{\nu}, w_{1}^{\lambda} w_{2}^{\mu} w_{3}^{\nu},
$$

where $\nu$ is the least number such that $\ldots w_{3}^{\nu}$ appears in $\gamma_{h}$ and $\mu$ is the least number such that $\ldots w_{2}^{\mu} w_{3}^{\nu}$ appears in $\gamma_{h}$. Let the coefficients of $w_{0}^{\lambda-i} w_{1}^{i} w_{2}^{\mu} w_{3}^{\nu}$ in $A$ be denoted by $a_{i}$. Then not all $a_{i}=0$. The coefficient of $w_{1}^{\lambda+1} w_{2}^{u} w_{3}^{\nu}$ in $\bar{E}$ is

$$
a_{0}(-X)^{\lambda}+a_{1}(-X)^{\lambda-1}+\ldots+a_{\lambda-1}(-X)+a_{\lambda} .
$$

This polynomial is not zero since $a_{i}$ are zero or polynomials of degree $m-2$ and not all $a_{i}=0$. Moreover the degree of this polynomial on $Z$ is not greater than $m-2$, therefore this polynomial is not divisible by $F$, which is a contradiction.

16) See A. Seidenberg [9]. 
Remark. An example shows that we can not omit the condition of characteristic 0 in Theorem 7 , but if the characteristic is greater than the square of the degree of $V$, then the theorem holds.

\section{REFERENCES}

[1] C. Chevalley, On the theory of local rings, Annals of Math., vol. 44 (1943).

[2] D. Gorenstein, An arithmetic theory of adjoint plane curves, Trans. Amer. Math. Soc. 72 (1952).

[ 3 ] E. Hecke, Vorlesungen über die Theorie der algebraischen Zahlen, (1923).

[4] J. Igusa, On the Picard varieties attached to algebraic varieties, Amer. J. Math. 74 (1952).

[5] H. W. E. Jung, Primteiler algebraischer Funktionen zweier unabhängigen Veränderlichen, Pal. Rend, vol. 26 (1908).

[6] S. Koizumi, On the differential forms of the first kind on algebraic varieties, Journ. Math. Soc. of Japan, vol. 1 (1949).

[7] Y. Nakai, On the divisors of differential forms on algebraic varieties, Journ. Math. Soc. of Japan, vol. 5 (1953).

[8] Y. Nakai, Some results in the theory of the differential forms of the first kind on algebraic varieties, Proc. Int. Symp. on Alg. Number Theory, (1956).

[9] A. Seidenberg, The hyperplane sections of normal varieties, Trans. Amer. Math. Soc. 69 (1950).

[10] F. Severi, Sugl'integrali algebrici semplici e doppi, Rend. della R. Acc. dei Lincei, s. VI, vol. VII (1928).

[11] A. Weil, Foundations of Algebraic Geometry, (1946).

[12] J. Weissinger, Zur arithmetische Theorie separierbarer Funktionenkörper, Abh. Math. Sem. Univ. Hamburg, vol. 16 (1948).

\section{Mathematical Institute}

Nagoya University 